\title{
Anisotropic damage mechanics for viscoelastic ice
}

\section{Journal Article}

Author(s):

Pralong, A.; Hutter, K.; Funk, M.

Publication date:

2006

Permanent link:

https://doi.org/10.3929/ethz-b-000001735

Rights / license:

In Copyright - Non-Commercial Use Permitted

Originally published in:

Continuum Mechanics and Thermodynamics 17(5), https://doi.org/10.1007/s00161-005-0002-5 


\title{
ORIGINAL ARTICLE
}

\author{
A. Pralong • K. Hutter • M. Funk
}

\section{Anisotropic damage mechanics for viscoelastic ice}

Received: 16 August 2005 / Accepted: 28 September 2005 / Published online: 3 February 2006

(C) Springer-Verlag 2005

\begin{abstract}
We present a formulation of continuum damage in glacier ice that incorporates the induced anisotropy of the damage effects but restricts these formally to orthotropy. Damage is modeled by a symmetric second rank tensor that structurally plays the role of an internal variable. It may be interpreted as a texture measure that quantifies the effective specific areas over which internal stresses can be transmitted. The evolution equation for the damage tensor is motivated in the reference configuration and pushed forward to the present configuration. A spatially objective constitutive form of the evolution equation for the damage tensor is obtained. The rheology of the damaged ice presumes no volume conservation. Its constitutive relations are derived from the free enthalpy and a dissipation potential, and extends the classical isotropic power law by elastic and damage tensor dependent terms. All constitutive relations are in conformity with the second law of thermodynamics.
\end{abstract}

Keywords Damage mechanics - Induced anisotropy · Constitutive relations · Viscoelasticity · Ice mechanics

PACS 83.60.Df, 62.20.Mk

\section{Introduction}

Glacier ice is ordinarily assumed to be an isotropic non-linearly viscous, incompressible medium and thus qualifies as a fluid. It is usually modeled by a Glen-type flow law [1]. Above a certain stress threshold, damage, interpreted as the deterioration of the material properties due to the formation of cracks, is observed in ice. Damage introduces a change of volume [2-4], and this violates the original assumption of incompressibility. Moreover, formation of cracks is a property typifying a solid. We will thus treat ice as a compressible solid with viscous and elastic properties, possibly anisotropic, because of the nonuniform orientation distribution of the cracks. When damage tends to vanish, ice will be considered as an incompressible viscoelastic solid. The elastic and damage effects will be introduced, such that, if they are ignored, formally a viscous isotropic fluid like material emerges and the classical Glen-type flow law can

Communicated by W.H. Müller

A. Pralong · M. Funk $(\varangle)$

Laboratory of Hydraulics, Hydrology and Glaciology, Swiss Federal Institute of Technology,

Gloriastr. 37-39, 8092 Zurich, Switzerland

E-mail: funk@vaw.baug.ethz.ch

K. Hutter

Department of Mechanics, Darmstadt University of Technology, Hochschulstr. 1, 64289 Darmstadt, Germany 
be restored. Once the constitutive relations for damaged ice are derived, elastic effects may be dropped as being negligible. With such a viewpoint, the observed formation of cracks can be modeled without conceptual conflict that a fluid can form cracks.

The failure of ice under creep at low deformation rates is due to the progressive accumulation of microcracks [5-7]. These microcracks reduce the internal areas over which stress can be transmitted and thus increase (at a given external load) the effective stress by an amount corresponding to the loss of this area. If cracks were spherical voids, this loss would be independent of orientation, and it would suffice to account for it by an enhancement factor. This is the situation in isotropic damage models (e.g., $[3,4,8,9,10])$. However, microcracks orient themselves according to the state of stress and thus give rise to anisotropic damage. Weiss and Gay [11] found microcrack anisotropy during creep to failure for uniaxial compression at $3 \mathrm{MPa}$ and $263 \mathrm{~K}$. They quantified the orientation of the microcracks by analyzing sample-cross sections in planes parallel to the axis of loading. The flow of glaciers or ice caps is affected by crevasses. Damage theory can also be applied to consider crevasses, which are assimilated to macrocracks. The damaged ice should be assumed to be anisotropic to consider the different crevasse patterns. Therefore, for both microscopic and macroscopic damage approaches, damage should be considered to be anisotropic. Damage, in this paper will be described by a second rank tensor variable for which an evolution equation, i.e., a balance law (without flux tensor) will be postulated. A critical step in this postulation is the choice of the objective time derivative of this damage tensor, which is not unique. This tensor appears in the theory as a linear mapping between the stress, interpreted as the traction per unit geometric area and the effective stress, interpreted as the traction per unit damaged area (geometric area minus void area). We will postulate that this mapping is formally the same in the reference and present configurations. This requirement will -as we shall see- fix the objective time derivative to be used when the balance law for the damage is postulated.

The above interpretation appears to be new, as is the thermodynamic setting that we employ. Parametrization of damage, on the other hand, is not new [3-10, 12].

\section{Notations and definitions}

In what follows, vectors are denoted by overhead arrows, second order tensors are underlined and fourth order tensors are doubly underlined. We use general tensor notation and employ the summation convention according to which summation is understood over doubly repeated indices that stand above and below. Covariant (contravariant) components are written as sub- (super-) scripts. Components of vectors and tensors in a basis of the reference configuration will be denoted by capital Latin indices $(A, B, C$, $\ldots)$, those in a basis of the present configuration will be denoted by lower case Latin indices $(i, j, k, \ldots)$.

Let $\vec{x}=\overrightarrow{\tilde{x}}(t, \vec{X})$ represent the position of the material point of the body in the present (i.e., time $t$ ) configuration relative to its position $\vec{X}$ in the corresponding reference configuration. Let $\underline{F}(t, \vec{X})=$ $(\partial \overrightarrow{\tilde{x}} / \partial \vec{X})(t, \vec{X})$ be the deformation gradient tensor, $\vec{v}(t, \vec{x})=(\partial \overrightarrow{\tilde{x}} / \partial t)(t, \vec{X}(t, \vec{x}))$ the spatial velocity vector, $\underline{L}(t, \vec{x})=(\partial \vec{v} / \partial \overrightarrow{\tilde{x}})(t, \vec{x}, \vec{X}(t, \vec{x}))$ the velocity gradient tensor, $\underline{d}=\frac{1}{2}\left(\underline{L}+\underline{L}^{T}\right)$ the stretching or strain rate tensor ${ }^{1}$ and $\underline{W}=\frac{1}{2}\left(\underline{L}-\underline{L}^{T}\right)$ the spin tensor. The scalar and dyadic products of two vectors $\vec{a}, \vec{b}$ will be denoted by $\vec{a} \cdot \vec{b}$ and $\vec{a} \otimes \vec{b}$, respectively, and the inner product of two symmetric second rank tensors $\underline{A}, \underline{B}$ is given by $\underline{A} \cdot \underline{B}=\operatorname{Tr}\left(\underline{A}^{T} \underline{B}\right)=\operatorname{Tr}\left(\underline{A}^{B} \underline{B}^{T}\right)$. Moreover, the Lie [,] and Jacobi $\langle$, brackets of two second rank tensors are defined by $[\underline{A}, \underline{B}]=\underline{A} \underline{B}-\underline{B} \underline{A}$ and $\langle\underline{A}, \underline{B}\rangle=\underline{A} \underline{B}+\underline{B} \underline{A}$, respectively. Formulations relative to the referential system are called Lagrangian, those in the present (spatial) description are denoted Eulerian.

\section{Continuum damage mechanics}

\subsection{Effective stress and damage effect tensor}

Let $\underline{\sigma}$ be the Cauchy stress tensor, i.e., the force in the present configuration, per damaged (ice plus voids) surface area. Let, moreover, the effective stress, i.e., the force in the present configuration, per

\footnotetext{
1 We use $\underline{d}$ instead of $\underline{D}$ because, later on, $\underline{D}$ will be reserved for damage.
} 
unit undamaged area (ice without voids) be $\underline{\tilde{\sigma}}$. In a linear mapping $\mathcal{L}: \underline{\sigma} \rightarrow \underline{\tilde{\sigma}}$, that accounts for the anisotropy, this yields

$$
\tilde{\sigma}^{i j}=Z^{i j}{ }_{k l} \sigma^{k l} .
$$

$\underline{Z}$ has the following properties: symmetry $k l \leftrightarrow l k$, since $\sigma$ is the symmetric and the pair symmetry $\overline{\overline{(i j}} j)(k l) \leftrightarrow(k l)(i j)$, which makes the mapping path independent; that is, $\underline{Z}$ can be derived from a potential. By assuming the symmetry of $\underline{\tilde{\sigma}}, \underline{Z}$ possesses also the symmetry $i j \leftrightarrow j i . \underline{Z}$ is finally postulated to be non-negative definite. The fourth order tensor $\underline{Z}$ is called the damage effect tensor. It is related to damage as will become apparent in the course of our study, and it allows for arbitrary induced anisotropy. However, in glaciology most models restrict themselves to orthotropy [13, 14] by choosing

$$
Z^{i j}{ }_{k l}=\frac{1}{2}\left(Z^{i}{ }_{k} \delta^{j}{ }_{l}+Z^{j}{ }_{k} \delta^{i}{ }_{l}\right) .
$$

From the pair symmetry of $\underline{Z}$, it follows that $\underline{Z}$ is symmetric, i.e., $Z^{i}{ }_{k}=Z_{k}{ }^{i}$. This orthotropic reduction yields for (3.1)

$$
\tilde{\sigma}^{i j}=\frac{1}{2}\left(Z^{i}{ }_{k} \sigma^{k j}+Z^{j}{ }_{k} \sigma^{k i}\right), \quad \underline{\tilde{\sigma}}=\frac{1}{2}\left(\underline{Z} \underline{\sigma}+(\underline{Z} \underline{\sigma})^{T}\right),
$$

where (.) $)^{T}$ denotes transposition.

\subsection{Balance laws}

Ice is known to be incompressible. However, because of the opening of the cracks, an adequate continuum model is likely not density preserving, ${ }^{2}$ so that the balance laws of mass, linear momentum and energy take the forms

$$
\begin{aligned}
\mathcal{M} & :=\dot{\rho}+\rho v^{i},{ }_{i}=0, \\
\mathcal{V}^{i} & :=\rho \dot{v}^{i}-\sigma^{i j},{ }_{j}-\rho b^{i}=0^{i}, \\
\mathcal{E} & :=\rho \dot{\varepsilon}+q^{i},{ }_{i}-\sigma^{i j} d_{i j}-\rho r=0,
\end{aligned}
$$

in which $\rho, v^{i}, \sigma^{i j}, b^{i}, \varepsilon, q^{i}, d_{i j}=\frac{1}{2}\left(v_{i},{ }_{j}+v_{j},{ }_{i}\right), r$ are the density, velocity, Cauchy stress, body force vector, internal energy, heat flux vector, stretching (strain rate) tensor and the enery supply rate density of the damaged ice.

In writing down the balance laws (3.4), it is implicitly assumed that the ice in focus is cold. With little formal change, a continuum damage model can also be developed for temperate ice. It would involve an additional mass balance equation for the moisture content, and the energy balance would serve as an equation determining the specific melting rate rather than the evolution of the temperature. We present here the theory for cold ice, because it is slightly simpler.

Equations (3.4) constitute 5 partial differential equations for the variables $\rho, \vec{v}$ and $\theta$. Here $\theta$ is the empirical temperature. So, quantities for which constitutive relations must be formulated are $\underline{\sigma}, \varepsilon$ and $\vec{q}$.

Since these constitutive quantities may depend on the damage, i.e., on the second order tensor $\underline{Z}$, the above balance laws must be complemented by an evolution equation for the damage tensor $\underline{Z}$, which we postulate to have internal variable structure

$$
\mathcal{Z}^{i}{ }_{j}:=\nabla^{i}{ }_{j}-f^{i}{ }_{j}=0^{i}{ }_{j},
$$

where ${ }^{\nabla}$ denotes a spatially objective time derivative. The symmetric tensor $f$ is a production term called the dynamic function of damage. A justification of (3.5) will be given below. Here it may suffice that (3.5) is a structural equation and therefore of constitutive nature.

2 For damaged ice, we do not use the term "incompressible" because the ice may still preserve its own density, whilst the composite "ice plus cracks" does not. 
For thermodynamic reasons, we also complement the balance laws (3.4) and (3.5) by an entropy inequality

$$
\mathcal{G}:=\rho \dot{\eta}+\phi^{i},{ }_{i}-\rho s \geq 0,
$$

where $\eta$ is the specific entropy, $\vec{\phi}$ its flux and $s$ its supply, in which $\eta$ and $\vec{\phi}$ are constitutive quantities, whilst $s$ is a source. Thus,

$$
\mathcal{C}=(\underline{\sigma}, \varepsilon, \vec{q}, \underline{f}, \eta, \vec{\phi}),
$$

are the constitutive quantities. They are assumed to depend on a number of independent constitutive variables (to be specified below in (4.1)).

\subsection{Damage evolution}

Let carets identify quantities referred to the reference configuration. If $\underline{\tilde{\sigma}}$ is the effective Cauchy stress, then $\underline{\tilde{\tilde{\sigma}}}$ is the effective second Piola-Kirchhoff stress. Since both are derived from surface tractions via Cauchy's lemma, they transform according to (e.g., [15])

$$
\underline{\tilde{\tilde{\sigma}}}=J \underline{F}^{-1} \underline{\tilde{\sigma}} \underline{F}^{-T}, \quad \hat{\tilde{\sigma}}^{A B}=J\left(F^{-1}\right)^{A}{ }_{i} \tilde{\sigma}^{i j}\left(F^{-1}\right)^{B}{ }_{j},
$$

where $J=\operatorname{det} \underline{F}$ and $\underline{F}^{-T}=\left(\underline{F}^{-1}\right)^{T}$. This shows that the "pull back" of doubly contravariant tensor components in the present configuration is accomplished by $\underline{F}^{-1}$ and $\underline{F}^{-T}$, respectively. Strains transform differently: their "pull back" operations are $\underline{F}^{T}$ and $\underline{F}$, respectively, since they are expressed as doubly covariant tensor components in the present configuration. Equation (3.2) clearly evidences $\underline{Z}$ to be mixed, contravariant-covariant. The following transformation rule is postulated to apply between $\underline{Z}, \underline{f}$ in the present and reference configurations

$$
\{\underline{\hat{Z}}, \underline{\hat{f}}\}=J^{\beta} \underline{F}^{-1}\{\underline{Z}, \underline{f}\} \underline{F}, \quad\left\{\hat{Z}^{A}{ }_{B}, \hat{f}^{A}{ }_{B}\right\}=J^{\beta}\left(F^{-1}\right)_{i}^{A}\left\{Z_{j}^{i}, f_{j}^{i}\right\} F^{j}{ }_{B},
$$

in which $\beta$ is yet a free exponent. This transformation leads to non-symmetric tensors $\underline{\hat{Z}}$ and $\hat{f}$.

We now postulate the materially objective evolution equation for the damage variable as follows

$$
\frac{\mathrm{d}}{\mathrm{d} t} \int_{V} \underline{\hat{z}} \mathrm{~d} V-\int_{V} \underline{\hat{f}} \mathrm{~d} V=\underline{0}
$$

valid for all body parts $V$. Localization then yields

$$
\frac{\mathrm{d}}{\mathrm{d} t} \underline{\hat{z}}-\underline{\hat{f}}=0
$$

With the transformation (3.9), the balance of damage in the Lagrangian description (see (3.11)) takes in the Eulerian description the form of (3.5), with the time derivative given by ${ }^{3}$

$$
\underline{\nabla}=\underline{\dot{Z}}+[\underline{Z}, \underline{W}]+\beta \underline{Z} \operatorname{div} \vec{v}
$$

where $\underline{W}$ is the spin tensor and the material derivative is given by

$$
\underline{\dot{Z}}=\frac{\partial \underline{Z}}{\partial t}+\operatorname{grad} \underline{Z} \cdot \vec{v}
$$

$\underline{\nabla}$ is spatially objective. With $\beta=0$, the time derivative (3.12) corresponds to the Jaumann derivative.

3 Since $\underline{\hat{Z}}$ and $\hat{f}$ are non-symmetric tensors, the time derivative derived from (3.9) and (3.11) is a non-symmetric tensor. To preserve the symmetry of $\underline{Z}$ (which has been postulated above), only the symmetric part of the time derivative is considered in (3.12). 
Remark 1 Let $Z^{i}{ }_{j}=Z \delta_{j}^{i}$, where $Z$ is a simple scalar, then (3.5) and (3.12) imply

$$
\begin{cases}\dot{Z}-f=0, & \text { if } \quad \beta=0, \\ \dot{Z}+Z \operatorname{div} \vec{v}-f=0, & \text { if } \quad \beta=1,\end{cases}
$$

as evolution equations for the scalar damage variable $Z$. They correspond to the global balances

$$
\begin{cases}\frac{\mathrm{d}}{\mathrm{d} t} \int_{v} \rho Z \mathrm{~d} v-\int_{v} \rho f \mathrm{~d} v=0, & \text { if } \quad \beta=0, \\ \frac{\mathrm{d}}{\mathrm{d} t} \int_{v} Z \mathrm{~d} v-\int_{v} f \mathrm{~d} v=0, & \text { if } \quad \beta=1 .\end{cases}
$$

Remark 2 The arbitrary mapping $\mathcal{L}: \underline{\tilde{\sigma}}=\underline{Z} \underline{\sigma}$ in the Eulerian description implies $\underline{\tilde{\tilde{\sigma}}}=J^{-\beta} \underline{\hat{Z}} \underline{\hat{\sigma}}$. Thus

$$
\underline{\tilde{\sigma}}=\underline{Z} \underline{\sigma} \rightarrow \underline{\hat{\tilde{\sigma}}}=\underline{\hat{Z}} \underline{\hat{\sigma}} \Longleftrightarrow \beta=0 .
$$

This implies that $\underline{Z}$ and $\underline{\hat{Z}}$ have the same properties in both configurations if $\beta=0$. So, the scalar damage variable $Z$ should be interpreted as a damage per unit mass according to $(3.14)_{1}$ and $(3.15)_{1}$. We shall see that the choice $\beta=0$ also has formal advantages.

The transformation properties stated in Remarks 1 and 2 can serve as motivation to choose the transformation rule (3.9) with $\beta=0$ as those defining the physical properties of the damage evolution equation. In the further developments, we will set $\beta=0$.

\section{Constitutive theory}

As stated earlier, constitutive relations are needed for the constitutive quantities $\mathcal{C}$ in (3.7). We require the constitutive quantities (3.7) to be functions of a set of independent constitutive variables ${ }^{4}$

$$
\mathcal{S}=(\hat{\rho}, \underline{B}, \theta, \underline{Z}, \vec{g}, \underline{d}) .
$$

A dependence on the left Cauchy-Green tensor $\underline{B}=\underline{F} \underline{F}^{T}$ accounts for the dependence of the equilibrium stress on strain. With the density $\hat{\rho}$ in the virgin (reference) configuration, it may automatically account for the density of damaged ice at equilibrium (which equals $\hat{\rho}(\operatorname{det} \underline{B})^{-\frac{1}{2}}$ ). $\theta$ and $\vec{g}=\operatorname{grad} \theta$ model thermal and heat conducting effects, $\underline{Z}$ accounts for the effects of the cracks on the material behavior and especially the anisotropy induced by them, and $\underline{d}$ describes viscous effects. In postulating (4.1), the ice is assumed to be cold.

The second law of thermodynamics requires the entropy inequality (3.6) to hold for all thermodynamic processes, i.e., those processes for which the balance laws (3.4) and (3.5) with $\mathcal{C}=\mathcal{C}(\mathcal{S})$ are satisfied. It follows from the work of Liu [16] that this requirement is satisfied if the inequality

$$
\rho \dot{\eta}+\operatorname{div} \vec{\phi}-\rho s-\Lambda^{\mathcal{M}} \mathcal{M}-\vec{\Lambda}^{\mathcal{V}} \cdot \overrightarrow{\mathcal{V}}-\Lambda^{\mathcal{E}} \mathcal{E}-\underline{\Lambda}^{\mathcal{Z}} \cdot \underline{\mathcal{Z}} \geq 0
$$

holds true for arbitrary smooth fields. In inequality (4.2), the mass, momentum and energy balances as well as the damage evolution equation are inserted in the inequality by multiplying the respective equations: $\mathcal{M}=0, \overrightarrow{\mathcal{V}}=\overrightarrow{0}, \mathcal{E}=0$ and $\underline{\mathcal{Z}}=\underline{0}$ with Lagrange multipliers $\Lambda^{\mathcal{M}}, \vec{\Lambda} \mathcal{V}, \Lambda^{\mathcal{E}}$ and $\underline{\Lambda}^{\mathcal{Z}}$, respectively. $\underline{\Lambda}^{\mathcal{Z}}$ is a symmetric tensor. The Lagrange multipliers are functions of the constitutive variables $\mathcal{S}$, of the velocity $\vec{v}$ and of the supply rate densities $r$ and $\vec{b}$. In the exploitation of the entropy inequality, the balance laws (3.4) and (3.5) serve as constraints. Since the constitutive relations $\mathcal{C}(\mathcal{S})$ cannot depend on $r$ and $\vec{b}$ and inequality (4.2) is a relation to constrain the constitutive relations, the external source terms in inequality (4.2) must add to zero, implying that

$$
-s+\vec{\Lambda}^{\mathcal{V}} \cdot \vec{b}+\Lambda^{\mathcal{E}} r=0 .
$$

\footnotetext{
4 The set (4.1) accounts for the fulfillment of the rule of material frame indifference.
} 
To exploit the imbalance (4.2), we introduce the generalized Gibbs relations by [17]

$$
\rho \mathrm{d} \eta=\Lambda^{\mathcal{E}} \rho \mathrm{d} \varepsilon+\mathrm{d} \mathcal{P} \quad \text { and } \quad \mathrm{d} \vec{\phi}=\Lambda^{\mathcal{E}} \mathrm{d} \vec{q}+\mathrm{d} \overrightarrow{\mathcal{F}}
$$

where ${ }^{5}$

$$
\begin{aligned}
& \mathrm{d} \mathcal{P}=\mathcal{P}_{\hat{\rho}} \mathrm{d} \hat{\rho}+\left(\mathcal{P}_{B}\right)_{i j} \mathrm{~d} B_{i j}+\mathcal{P}_{\theta} \mathrm{d} \theta+\left(\mathcal{P}_{Z}\right)_{i j} \mathrm{~d} Z_{i j}+\left(\mathcal{P}_{g}\right)_{i} \mathrm{~d} g_{i}+\left(\mathcal{P}_{d}\right)_{i j} \mathrm{~d} d_{i j}, \\
& \mathrm{~d} \mathcal{F}_{i}=\left(\mathcal{F}_{\hat{\rho}}\right)_{i} \mathrm{~d} \hat{\rho}+\left(\mathcal{F}_{B}\right)_{i j k} \mathrm{~d} B_{j k}+\left(\mathcal{F}_{\theta}\right)_{i} \mathrm{~d} \theta+\left(\mathcal{F}_{Z}\right)_{i j k} \mathrm{~d} Z_{j k}+\left(\mathcal{F}_{g}\right)_{i j} \mathrm{~d} g_{j}+\left(\mathcal{F}_{d}\right)_{i j k} \mathrm{~d} d_{j k} .
\end{aligned}
$$

In particular, $\underline{\mathcal{P}}_{B}$ and $\underline{\mathcal{P}}_{Z}$ are symmetric tensors. Substituting (3.4), (3.5) and (4.4) into inequality (4.2) and accounting for (4.3), results in a new inequality that can be written in the following form $^{6}$

$$
\vec{\alpha}(\mathcal{S}) \cdot \vec{a}+\pi(\mathcal{S}) \geq 0 \quad \forall \vec{a} .
$$

$\vec{\alpha}$ and $\vec{a}$ are vectors formed by scalars and components of vectors and tensors. Also $\vec{a}$ does not depend on the variables forming the set $\mathcal{S}$ but on the spin tensor and certain higher space and time derivatives of $\vec{v}$, $\underline{\sigma}$ and $\mathcal{S} . \pi(\mathcal{S})$ is the so-called residual entropy production. It reads

$$
\pi(\mathcal{S})=\left(\left\langle\underline{\mathcal{P}}_{B}, \underline{B}\right\rangle+\Lambda^{\mathcal{E}} \underline{\sigma}+\Lambda^{\mathcal{M}} \hat{\rho}(\operatorname{det} \underline{B})^{-\frac{1}{2}} \underline{I}-\vec{g} \otimes \overrightarrow{\mathcal{P}}_{g}\right) \cdot \underline{d}+\overrightarrow{\mathcal{F}}_{\theta} \cdot \vec{g}+\vec{\Lambda}^{\mathcal{V}} \cdot \operatorname{div} \underline{\sigma}+\underline{\Lambda}^{\mathcal{Z}} \cdot \underline{f},
$$

where $\underline{I}$ is the second order unit tensor. Evidently, inequality (4.6) is linear in $\vec{a}$, and since $\vec{a}$ may have any arbitrarily assigned value, it would be violated unless

$$
\vec{\alpha}(\mathcal{S})=\overrightarrow{0} \quad \text { and } \quad \pi(\mathcal{S}) \geq 0 .
$$

The identities $(4.8)_{1}$ constitute relations between $(\eta, \varepsilon),(\vec{\phi}, \vec{q})$ and the Lagrange multipliers $\vec{\Lambda}^{\mathcal{V}}$ and $\underline{\Lambda}^{\mathcal{Z}}$. From them we may deduce

$$
\Lambda^{\mathcal{M}}=0, \quad \vec{\Lambda}^{\mathcal{V}}=\overrightarrow{0} \quad \text { and } \quad \underline{\Lambda}^{\mathcal{Z}}=\underline{\mathcal{P}}_{Z} .
$$

Moreover,

Finally, $\mathrm{d} \mathcal{P}$ and $\mathrm{d} \overrightarrow{\mathcal{F}}$ reduce to

$$
\left[\underline{\mathcal{P}}_{Z}, \underline{Z}\right]+\left[\underline{\mathcal{P}}_{B}, \underline{B}\right]=\underline{0} .
$$

$$
\mathrm{d} \mathcal{P}=\underline{\mathcal{P}}_{B} \cdot \mathrm{d} \underline{B}+\underline{\mathcal{P}}_{Z} \cdot \mathrm{d} \underline{Z} \quad \text { and } \quad \mathrm{d} \overrightarrow{\mathcal{F}}=\overrightarrow{\mathcal{F}}_{\theta} \mathrm{d} \theta .
$$

According to the "differential" approach by Liu [19] (see also [17]) and since $\mathcal{C}(\mathcal{S})$ are isotropic functions of their arguments, ${ }^{7}$ it follows that

$$
\vec{\phi}=\Lambda^{\mathcal{E}} \vec{q} \quad \text { and } \quad \Lambda^{\mathcal{E}}=\Lambda^{\mathcal{E}}(\theta) .
$$

It can be shown that (e.g., [18])

$$
\Lambda^{\mathcal{E}}(\theta)=\frac{1}{\theta} .
$$

This identifies $\theta$ with the absolute temperature. So, the classical equation "entropy flux equals heat flux divided by absolute temperature" has been deduced. With (4.3) and (4.9) 2 , the relation "entropy source equals heat source divided by absolute temperature" can also be established.

\footnotetext{
5 Since we restrict ourselves to Cartesian tensors, we do no longer differentiate between upper and lower indices.

6 We only sketch the ensuing computations, but do not show the heavy details, since these computations are relatively routine for specialists and uninteresting for others. About exactly the situation dealt with here, we know of no explicit reference, but a paper close to this is [17], and analogous computations have also been done in [18], chap. 7. Whereas the derivation of the results is technical, they are important to guarantee a consistent thermodynamic model, even when temperature will eventually be left out of consideration.

7 All constitutive quantities (3.7) and constitutive variables (4.1) are objective under Euclidean transformations, so if $Q$ is an orthogonal transformation, then $Q^{*} \mathcal{C}(\underline{B}, \theta, \underline{Z}, \vec{g}, \underline{d})=\mathcal{C}\left(\underline{Q} \underline{B} \underline{Q}^{T}, \theta, \underline{Q} \underline{Z} \underline{Q}^{T}, \underline{Q} \vec{g}, \underline{Q} \underline{d} \underline{Q}^{T}\right)$, where $Q^{*}$ is the (push forward) action of $Q$ on $\mathcal{C}$. The fact that $\mathcal{C}(\mathcal{S})$ is taken as an isotropic function set does not imply that effects of anisotropy are absent. These are accounted for by $\underline{Z}$.
} 
With $(4.4)_{2},(4.11)_{2},(4.12)$ and (4.13) there follows

$$
\overrightarrow{\mathcal{F}}_{\theta}=-\vec{q} \frac{1}{\theta^{2}}
$$

Moreover, from relations $(4.4)_{1},(4.11)_{1}$ and (4.12) 2 one may deduce that $\eta$ and $\varepsilon$ cannot depend on $\vec{g}$ and $\underline{d}$, but only on $\underline{B}, \theta, \underline{Z}$; explicitly,

$$
\eta=\eta(\underline{B}, \theta, \underline{Z}), \quad \varepsilon=\varepsilon(\underline{B}, \theta, \underline{Z}) .
$$

Equations (4.4) $)_{1}$ and (4.15) imply that $\underline{\mathcal{P}}_{B}$ and $\underline{\mathcal{P}}_{Z}$ are equally functions of only $\underline{B}, \theta, \underline{Z}$. In fact, as evident from (4.4) $)_{1} \underline{\mathcal{P}}_{B}$ and $\underline{\mathcal{P}}_{Z}$ play the role of thermal equations of state that can be derived from the Helmholtz free energy

$$
\psi=\varepsilon-\theta \eta=\psi(\underline{B}, \theta, \underline{Z}) .
$$

Indeed, if $\varepsilon$ is eliminated between (4.4) $)_{1}$ and (4.16), the Gibbs relation (4.4) (with (4.11) 1 ) is identically satisfied, if

$$
\frac{\partial \psi}{\partial \underline{B}}=-\frac{\theta}{\rho} \underline{\mathcal{P}}_{B}, \quad \frac{\partial \psi}{\partial \theta}=-\eta, \quad \frac{\partial \psi}{\partial \underline{Z}}=-\frac{\theta}{\rho} \underline{\mathcal{P}}_{Z}
$$

Thus $\psi$ is a thermodynamic potential for $-\frac{\theta}{\rho} \underline{\mathcal{P}}_{B},-\eta$ and $-\frac{\theta}{\rho} \underline{\mathcal{P}}_{Z}$. If we define

$$
\underline{\Sigma}:=\frac{\partial \psi}{\partial \underline{B}}=-\frac{\theta}{\rho} \underline{\mathcal{P}}_{B}, \quad \underline{Y}:=\frac{\partial \psi}{\partial \underline{Z}}=-\frac{\theta}{\rho} \underline{\mathcal{P}}_{Z}
$$

with $\underline{\Sigma}=\underline{\Sigma}(\underline{B}, \theta, \underline{Z})$ and $\underline{Y}=\underline{Y}(\underline{B}, \theta, \underline{Z})$, then the Gibbs relation takes the form

$$
\mathrm{d} \eta=\frac{1}{\theta}(\mathrm{d} \varepsilon-\underline{\Sigma} \cdot \mathrm{d} \underline{B}-\underline{Y} \cdot \mathrm{d} \underline{Z}) .
$$

In this form, $\underline{\Sigma}$ is, clearly, a stress exhibiting elastic properties and $\underline{Y}$ is the tensorial energy release rate, the conjugate force (a symmetric tensor) to the damage variable $\underline{Z} .^{\bar{\gamma}}$

The Helmholtz free energy $\psi$ is an isotropic function of a scalar $\theta$ and two symmetric tensors, $\underline{B}$ and $\underline{Z}$, of which a complete set of invariants is given by $I_{\underline{A}_{\alpha}}, I_{\underline{A}_{\alpha}}, I I_{\underline{A}_{\alpha}}$, with

$$
I_{A}=\operatorname{Tr} \underline{A}, \quad I_{A}=\frac{1}{2}\left((\operatorname{Tr} \underline{A})^{2}-\operatorname{Tr}\left(\underline{A}^{2}\right)\right), \quad I I I_{A}=\operatorname{det} \underline{A}
$$

and

$$
\underline{A}_{1}=\underline{B}, \quad \underline{A}_{2}=\underline{Z}, \quad \underline{A}_{3}=\langle\underline{B}, \underline{Z}\rangle, \quad \underline{A}_{4}=\left\langle\underline{B}^{2}, \underline{Z}\right\rangle, \quad \underline{A}_{5}=\left\langle\underline{B}, \underline{Z}^{2}\right\rangle
$$

that is,

$$
\psi=\psi\left(\theta, I_{\underline{A}_{\alpha}}, I_{\underline{A}_{\alpha}}, I I I_{\underline{A}_{\alpha}}\right) .
$$

Once a functional relation for $\psi$ is selected, $\underline{\mathcal{P}}_{B}$ and $\underline{\mathcal{P}}_{Z}$ are known and must satisfy (4.10). With this, the exploitation of $(4.8)_{1}$ is now complete.

${ }^{8} \underline{Y}$ denotes in the literature the conjugate force of the damage variable $\underline{D}$ (see Sect. 7.4). We would like to call attention that $\underline{Y}$ is here conjugate to the damage effect variable $\underline{Z}$. 


\section{Thermodynamic equilibrium}

The next step is to exploit the residual entropy inequality (4.8) 2 . With (4.7), (4.9), (4.11), (4.13), (4.14) and (4.18), it becomes

$$
\theta \pi(\mathcal{S})=(-\rho\langle\underline{\Sigma}, \underline{B}\rangle+\underline{\sigma}) \cdot \underline{d}-\frac{\vec{q} \cdot \vec{g}}{\theta}-\rho \underline{Y} \cdot \underline{f} \geq 0 .
$$

The results to be derived below are based on the conditions of thermodynamic equilibrium. We therefore assume the existence of a thermodynamic equilibrium. If $\vec{e}=(\hat{\rho}, \underline{B}, \theta, \underline{Z})$ and $\vec{n}=(\vec{g}, \underline{d})$, then any dependent constitutive quantity $\mathcal{C}$ can be represented in the form

$$
\mathcal{C}(\mathcal{S})=\mathcal{C}(\vec{e}, \vec{n})=\left.\mathcal{C}\right|_{E}(\vec{e})+\left.\mathcal{C}\right|_{N}(\vec{e}, \vec{n})
$$

with $\left.\mathcal{C}\right|_{E}(\vec{e})=\mathcal{C}(\vec{e}, \vec{n}=\overrightarrow{0})$ and $\left.\mathcal{C}\right|_{N}(\vec{e}, \vec{n})=\mathcal{C}(\vec{e}, \vec{n})-\left.\mathcal{C}\right|_{E}(\vec{e})$ its equilibrium and non-equilibrium parts, respectively. $\left.\pi\right|_{E}=0$ must necessarily hold, for otherwise entropy would be produced in thermodynamic equilibrium. Therefore, from (5.1),

$$
\left.\underline{f}\right|_{E}=\underline{0} .
$$

An isotropic expansion of $\underline{f}(\mathcal{S})$ following the superposition principle by Betten [20] yields

$$
\begin{aligned}
\underline{f}= & \varphi_{0} \underline{I}+\varphi_{1 \alpha} \underline{A}_{\alpha}+\varphi_{2 \alpha} \underline{A}_{\alpha}^{2} \\
& +\varphi_{1 \alpha \beta}\left\langle\underline{A}_{\alpha}, \underline{A}_{\beta}\right\rangle+\varphi_{2 \alpha \beta}\left\langle\underline{A}_{\alpha}^{2}, \underline{A}_{\beta}\right\rangle+\varphi_{3 \alpha \beta}\left\langle\underline{A}_{\alpha}, \underline{A}_{\beta}^{2}\right\rangle \\
& +\varphi_{4 \alpha \beta}\left\langle\underline{A}_{\alpha}^{2}, \underline{A}_{\beta}^{2}\right\rangle+\varphi_{3 \alpha}\left\langle\vec{g} \otimes \vec{g}, \underline{A}_{\alpha}\right\rangle+\varphi_{4 \alpha}\left\langle\vec{g} \otimes \vec{g}, \underline{A}_{\alpha}^{2}\right\rangle,
\end{aligned}
$$

in which $\underline{I}$ is the second order unit tensor and summation is over $\alpha, \beta=1,2,3, \alpha>\beta$ and $\underline{A}_{1}=\underline{B}$, $\underline{A}_{2}=\underline{Z}, \underline{A}_{3}=\underline{d}$. The equilibrium part is given by

$$
\begin{aligned}
\left.\underline{f}\right|_{E}= & \left.\varphi_{0}\right|_{E} \underline{I}+\left.\varphi_{11}\right|_{E} \underline{B}+\left.\varphi_{12}\right|_{E} \underline{Z} \\
& +\left.\varphi_{21}\right|_{E} \underline{B}^{2}+\left.\varphi_{22}\right|_{E} \underline{Z}^{2}+\left.\varphi_{112}\right|_{E}\langle\underline{B}, \underline{Z}\rangle \\
& +\left.\varphi_{212}\right|_{E}\left\langle\underline{B}^{2}, \underline{Z}\right\rangle+\left.\varphi_{312}\right|_{E}\left\langle\underline{B}, \underline{Z}^{2}\right\rangle+\left.\varphi_{412}\right|_{E}\left\langle\underline{B}^{2}, \underline{Z}^{2}\right\rangle .
\end{aligned}
$$

For this to vanish at equilibrium, the restrictions

$$
\begin{aligned}
& \left.\varphi_{0}\right|_{E}=0,\left.\quad \varphi_{11}\right|_{E}=0,\left.\quad \varphi_{12}\right|_{E}=0,\left.\quad \varphi_{21}\right|_{E}=0,\left.\quad \varphi_{22}\right|_{E}=0, \\
& \left.\varphi_{112}\right|_{E}=0,\left.\quad \varphi_{212}\right|_{E}=0,\left.\quad \varphi_{312}\right|_{E}=0,\left.\quad \varphi_{412}\right|_{E}=0,
\end{aligned}
$$

must identically hold for consistent modeling. In general, the scalar coefficients in (5.4) are functions of the scalar invariants of $\mathcal{S}$. Notice that the dependence in these, as well as (5.4), is quadratic in $\vec{g}$. Since $\pi=\pi(\mathcal{S})$, its representation in terms of the invariants of $\mathcal{S}$ is also quadratic in $\vec{g}$. This implies, in particular that

$$
\left.\frac{\partial \pi}{\partial \vec{g}}\right|_{E}=\overrightarrow{0}, \quad \text { and }\left.\quad \frac{\partial^{2} \pi}{\partial \vec{g} \partial \underline{d}}\right|_{E}=0^{(3)},
$$

with $0^{(3)}$ the zero third order tensor.

Now consider once more the imbalance (5.1). Because $\pi(\vec{e}, \vec{n})$ assumes its minimum value, namely 0 , at $\vec{n}=\overrightarrow{0}$, necessary conditions to guarantee this minimum are

$$
\left.\frac{\partial \pi}{\partial \vec{g}}\right|_{E}=\overrightarrow{0},\left.\quad \frac{\partial \pi}{\partial \underline{d}}\right|_{E}=\underline{0},
$$


and, by simplifying with the aid of (5.7) 2

$$
H=\left.\left(\begin{array}{cc}
\partial^{2} \pi / \partial \vec{g} \partial \vec{g} & 0 \\
0 & \partial^{2} \pi / \partial \underline{d} \partial \underline{d}
\end{array}\right)\right|_{E} \text { is non-negative definite. }
$$

The first of (5.8) is fulfilled because of (5.7) $)_{1}$.

Next, consider $(5.8)_{2}$; it implies

$$
\left(\left.\sigma\right|_{E}\right)_{i j}=\rho\left\langle\Sigma_{i k}, B_{k j}\right\rangle+\left.\rho Y_{k l} \frac{\partial f_{k l}}{\partial d_{i j}}\right|_{E} .
$$

The first term on the right-hand side is the elastic contribution and accounts for the fact that the cracks may yield the material to become compressible, even though the virgin material may be incompressible. The second term on the right-hand side accounts for anisotropic equilibrium stresses due to damage.

Remark 3 If we let $\psi$ depend only on the determinant of $\underline{B}$ and define

$$
\psi=\psi_{1}(r)=\psi_{2}(\rho), \quad r=\frac{\hat{\rho}}{\rho}=(\operatorname{det} \underline{B})^{\frac{1}{2}},
$$

by using $\partial \operatorname{det} \underline{B} / \partial \underline{B}=(\operatorname{det} \underline{B}) \underline{B}^{-T}$ it is straightforward to show that

$$
\rho\left\langle\Sigma_{i k}, B_{k j}\right\rangle=\left\langle\rho \frac{\partial \psi}{\partial B_{i k}}, B_{k j}\right\rangle=-\rho^{2} \frac{\partial \psi_{2}}{\partial \rho} \delta_{i j}=-p_{\text {thermo }} \delta_{i j},
$$

in which $p_{\text {thermo }}$ agrees with the thermodynamic pressure. Therefore, the first term of the equilibrium stress (5.10) would be the thermodynamic pressure tensor. Similarly one may show that in this case $\underline{\mathcal{P}}_{B} \cdot \mathrm{d} \underline{B}$ in (4.5) would be

$$
\underline{\mathcal{P}}_{B} \cdot \mathrm{d} \underline{B}=\rho p_{\text {thermo }} \mathrm{d}\left(\frac{1}{\rho}\right),
$$

as would be expected if the material in its damaged state possesses properties of an elastic fluid.

With (5.10), we may introduce the irreversible stress $\left.\underline{\sigma}\right|_{N}$ according to (5.2). Note that $\left.\left(\left.\underline{\sigma}\right|_{N}\right)\right|_{E}=\underline{0}$. With (5.10), the residual entropy inequality (5.1) takes the form

$$
\theta \pi(\mathcal{S})=\left(\left.\sigma\right|_{N}\right)_{i j} d_{i j}-\frac{q_{i} g_{i}}{\theta}-\rho Y_{i j}\left(f_{i j}-\left.\frac{\partial f_{i j}}{\partial d_{k l}}\right|_{E} d_{k l}\right) \geq 0
$$

\section{Free enthalpy formulation}

For glaciologists, it is more common to express the deformation as a function of the stress. Therefore, we consider the deformation, $\underline{B}$ and $\underline{d}$ as dependent and $\underline{\Sigma}$ and $\left.\underline{\sigma}\right|_{N}$ as independent constitutive variables. Thus, (4.1) is replaced by

$$
\mathcal{S}_{G}=\left(\hat{\rho}, \underline{\Sigma}, \theta, \underline{Z}, \vec{g},\left.\underline{\sigma}\right|_{N}\right) .
$$

Introducing the free enthalpy function

$$
G=\varepsilon-\theta \eta-\underline{\Sigma} \cdot \underline{B}=G(\underline{\Sigma}, \theta, \underline{Z}),
$$

the Gibbs relation (4.19) is then identically satisfied, if

$$
\frac{\partial G}{\partial \underline{\Sigma}}=-\underline{B}, \quad \frac{\partial G}{\partial \theta}=-\eta, \quad \frac{\partial G}{\partial \underline{Z}}=\underline{Y},
$$


with $\underline{B}=\underline{B}(\underline{\Sigma}, \theta, \underline{Z})$ and $\underline{Y}=\underline{Y}(\underline{\Sigma}, \theta, \underline{Z})$. Furthermore, by accounting for (5.2), the entropy inequality (5.1) takes the form

$$
\theta \pi(\mathcal{S})=\left(-\rho\langle\underline{\Sigma}, \underline{B}\rangle+\left.\underline{\sigma}\right|_{N}+\left.\underline{\sigma}\right|_{E}\right) \cdot \underline{d}-\frac{\vec{q} \cdot \vec{g}}{\theta}-\rho \underline{Y} \cdot \underline{f} \geq 0 .
$$

If we let now $\vec{e}=(\hat{\rho}, \underline{\Sigma}, \theta, \underline{Z})$ and $\vec{n}=\left(\vec{g},\left.\underline{\sigma}\right|_{N}\right)$, then, according to the definition of thermodynamic equilibrium (5.2), $\left.\pi\right|_{E}=0$ must hold. Therefore, from (6.4),

$$
\left.\underline{d}\right|_{E}=\underline{0} \quad \text { and }\left.\quad \underline{f}\right|_{E}=\underline{0} .
$$

This then yields relations for $\underline{d}$ and $f$ as tensorial isotropic functions analogous to (5.4), (5.5) and (5.6), in which the variables $\underline{B}$ and $\underline{\underline{d}}$ are replaced by $\underline{\Sigma}$ and $\left.\underline{\sigma}\right|_{N}$, respectively. We refrain from writing them down explicitly. Since, moreover, $\pi$ assumes its minimum in equilibrium, we necessarily have

$$
\left.\frac{\partial \pi}{\partial \vec{g}}\right|_{E}=\overrightarrow{0},\left.\quad \frac{\partial \pi}{\left.\partial \underline{\sigma}\right|_{N}}\right|_{E}=\underline{0}
$$

and

$$
H=\left.\left(\begin{array}{cc}
\partial^{2} \pi / \partial \vec{g} \partial \vec{g} & 0 \\
0 & \partial^{2} \pi /\left.\left.\partial \underline{\sigma}\right|_{N} \partial \underline{\sigma}\right|_{N}
\end{array}\right)\right|_{E} \text { is non-negative definite. }
$$

Since $\pi$ is quadratic in $\vec{g},(6.6)_{1}$ is automatically fulfilled. Alternatively, with $(6.5)_{1},(6.6)_{2}$ may be written as

$$
\left.\left[\left(\left.\sigma\right|_{E}\right)_{k l}-\rho\left\langle\Sigma_{k m}, B_{m l}\right\rangle\right] \frac{\partial d_{k l}}{\partial\left(\left.\sigma\right|_{N}\right)_{i j}}\right|_{E}-\left.\rho Y_{k l} \frac{\partial f_{k l}}{\partial\left(\left.\sigma\right|_{N}\right)_{i j}}\right|_{E}=0 .
$$

If the six matrices $\left.\left(\partial d_{k l} / \partial\left(\left.\sigma\right|_{N}\right)_{i j}\right)\right|_{E}(k, l=1,2,3$ are fixed and $k \leq l)$ are invertible in the sense that

$$
\left.\left.\frac{\partial d_{k l}}{\partial\left(\left.\sigma\right|_{N}\right)_{i j}}\right|_{E} \frac{\partial\left(\left.\sigma\right|_{N}\right)_{i j}}{\partial d_{p q}}\right|_{E}=\delta_{k p} \delta_{l q}
$$

then (6.8) becomes

$$
\left(\left.\sigma\right|_{E}\right)_{p q}=\rho\left\langle\Sigma_{p m}, B_{m q}\right\rangle+\left.\left.\rho Y_{k l} \frac{\partial f_{k l}}{\partial\left(\left.\sigma\right|_{N}\right)_{i j}}\right|_{E} \frac{\partial\left(\left.\sigma\right|_{N}\right)_{i j}}{\partial d_{p q}}\right|_{E} .
$$

which corresponds to (5.10) in the earlier formulation. Finally, the entropy inequality in the "enthalpy" formulation now takes the form

$$
\theta \pi(\mathcal{S})=\left(\left.\sigma\right|_{N}\right)_{i j} d_{i j}-\frac{q_{i} g_{i}}{\theta}-\rho Y_{i j}\left(f_{i j}-\left.\left.\frac{\partial f_{i j}}{\partial\left(\left.\sigma\right|_{N}\right)_{k l}}\right|_{E} \frac{\partial\left(\left.\sigma\right|_{N}\right)_{k l}}{\partial d_{p q}}\right|_{E} d_{p q}\right) \geq 0 .
$$

which is analogous to inequality (5.14). This completes the thermodynamic analysis in the free energy and enthalpy formulations.

\section{Damage model for ice}

The proposed damage model for ice will be expressed in the enthalpy formulation and so constitutive quantities are functions of $\mathcal{S}_{\mathrm{G}}$. What remains is to propose explicit expressions for the heat flux $\vec{q}$, the free enthalpy $G$, the dissipative strain rate $\underline{d}$ and the dynamic function of damage $\underline{f}$. 


\subsection{Heat flux}

$\vec{q}$ is assumed to depend on $\mathcal{S}_{G}$ according to the Fourier law of heat conduction

$$
\vec{q}=-\underline{\kappa}\left(\mathcal{S}_{G}\right) \vec{g}, \quad \text { with } \underline{\kappa}\left(\mathcal{S}_{G}\right) \text { positive definite. }
$$

$\underline{\kappa}$ is the heat conductivity. For ice, it is reasonable to restrict the function $\underline{\kappa}=\underline{\kappa}\left(\mathcal{S}_{G}\right)$ to $\underline{\kappa}=\underline{\kappa}(\rho, \theta, \underline{Z})$. According to (4.12) 1 and (4.13), the entropy flux becomes then

$$
\vec{\phi}=-\frac{1}{\theta} \kappa \vec{g}
$$

\subsection{Free enthalpy}

Let us assume for undamaged ice at thermodynamic equilibrium a linear dependence between $(\underline{B}-\underline{I})$ and $\underline{\Sigma}$ of the form

$$
\Sigma_{i j}=A_{i j k l}\left(B_{k l}-I_{k l}\right),
$$

with $\underline{\underline{A}}$ a stiffness tensor. Assume, moreover, stability of relation (7.3); that is, $\underline{\underline{A}}$ is positive definite

$$
A_{i j k l}\left(B_{i j}-I_{i j}\right)\left(B_{k l}-I_{k l}\right)>0 \quad \forall\left(B_{i j}-I_{i j}\right) \neq 0 .
$$

By considering (6.2) and neglecting thermoelastic effects (i.e., coupling), the free enthalpy $G$ for linear and initially isotropic material at equilibrium will be postulated in the form (see also [21])

$$
G(\underline{\Sigma}, \theta, \underline{Z})=-\frac{1+a_{2}}{2 a_{1}} I_{\underline{Z}\left(\underline{\Sigma}^{D}\right)^{2}}-\frac{1-2 a_{2}}{2 a_{1}} \xi I_{\underline{Z}} I_{\underline{\Sigma}}^{2}-I_{\underline{\Sigma}}-\int_{\theta_{0}}^{\theta} G^{\theta}(\vartheta) \mathrm{d} \vartheta,
$$

with $a_{1}$ and $a_{2}$ the only two parameters characterizing $\underline{\underline{A}}$ for isotropic undamaged material, $z>0$ a parameter similar to that introduced by Lemaitre et al. $[2 \overline{1}]$ to differentiate the effect of damage on the deviatoric and the hydrostatic parts of the free enthalpy (the first and the second terms, respectively, on the right-hand side of (7.5)), $G^{\theta}(\theta)$ a thermal function and $\theta_{0}$ a reference temperature. The superscript ()$^{D}$ refers to the deviatoric part of a second order tensor. The first three terms on the right-hand side describe the elastic deformation and the forth term accounts for the thermal behavior of ice. With (6.3) 1 , $\underline{B}$ becomes

$$
\underline{B}=\frac{1+a_{2}}{a_{1}} \underline{\tilde{\Sigma}}^{D}-\frac{a_{2}}{a_{1}} I_{\tilde{\Sigma}^{D}} \underline{I}+\underline{I},
$$

where ${ }^{9}$

$$
\underline{\tilde{\Sigma}}^{D}=\frac{1}{2}\left\langle\underline{Z}^{T}, \underline{\Sigma}^{D}\right\rangle^{D}+\xi I_{\underline{Z}} I_{\underline{\Sigma}} \underline{I} .
$$

Considering $(6.3)_{3}$, the tensorial energy release rate $\underline{Y}$ reads

$$
\underline{Y}=-\frac{1+a_{2}}{2 a_{1}}\left(\underline{\Sigma}^{D}\right)^{2}-\frac{1-2 a_{2}}{2 a_{1}} \xi I_{\underline{\Sigma}}^{2} \underline{I} .
$$

Using (4.18), (7.6) and (7.8), it can be shown that the free enthalpy as proposed in (7.5) automatically satisfies the thermodynamic condition (4.10). For isotropic undamaged material, by assuming $a_{1}>0$, the stability condition (7.4) reduces to the requirements

$$
\left(1+a_{2}\right)>0 \text { and }\left(1-2 a_{2}\right)>0 .
$$

\footnotetext{
9 By deriving $B$ from $G$, the transpose of $Z$ appears in relation (7.7). Since $\Sigma$ and $\tilde{\Sigma}$ are covariant tensors, it can be observed in (7.7) that $\underline{Z}$ is contravariant-covariant. Therefore the objective time derivative (3.12), i.e., the whole thermodynamic analysis, is also valid for the damage tensor $\underline{Z}$ as introduced in the free enthalpy. Since we restrict ourselves to Cartesian coordinates, we do no longer differentiate between $\underline{Z}$ and $\underline{Z}^{T}$.
} 
Therefore, using (7.8), one can show that

$$
\underline{Y} \text { is negative definite. }
$$

This is an important result, which will be discussed later when a damage production rate function $\underline{f}$ will be proposed.

According to $(6.3)_{2}$, the entropy takes the form

$$
\eta=G^{\theta}(\theta)
$$

Let us now express the purely thermal part $G^{\theta}(\theta)$ of the free enthalpy as

$$
G^{\theta}(\theta)=c_{v} \ln \left(\frac{\theta}{\theta_{0}}\right)+G_{0}^{\theta},
$$

with $c_{v}$ the specific heat at constant volume and $G_{0}^{\theta}$ a constant. With the aid of (6.2), (7.5), (7.6) and (7.11), the internal energy becomes

$$
\varepsilon=\varepsilon_{\mathrm{el}}+\varepsilon_{\mathrm{th}}
$$

with $\varepsilon_{\mathrm{el}}$ and $\varepsilon_{\mathrm{th}}$ the mechanical and thermal parts of $\varepsilon$ expressed as

$$
\varepsilon_{\mathrm{el}}=\frac{1+a_{2}}{2 a_{1}} I_{\underline{Z}\left(\underline{\Sigma}^{D}\right)^{2}} \text { and } \quad \varepsilon_{\mathrm{th}}=c_{v}\left(\theta-\theta_{0}\right)+G_{0}^{\theta} \theta_{0} .
$$

\subsection{Dissipative strain rate}

Let us assume a dissipation potential $\varphi\left(\mathcal{S}_{G}\right)$ for the dissipative stress $\left.\underline{\sigma}\right|_{N}$, with the property that

$$
\underline{d}=\frac{\partial \varphi\left(\mathcal{S}_{G}\right)}{\left.\partial \underline{\sigma}\right|_{N}} .
$$

Assuming that $\varphi$ is a function of $\theta, \underline{Z}$ and $\left.\underline{\sigma}\right|_{N}$ only and omitting explicit terms in $\underline{Z}^{2},\left(\left.\underline{\sigma}\right|_{N}\right)^{2}$ and of higher order, (7.15) can be shown to take the form

$$
\begin{aligned}
\underline{d}= & \left(\delta_{11}+\delta_{12} I_{\left.\left.\underline{\sigma}\right|_{N}\right)} \underline{I}-\left.\delta_{12} \underline{\sigma}\right|_{N}+\delta_{13} I I I_{\left.\underline{\sigma}\right|_{N}}\left(\left.\underline{\sigma}\right|_{N}\right)^{-1}\right. \\
& +2\left(\delta_{21}+\delta_{22} I_{\left\langle\underline{Z},\left.\underline{\sigma}\right|_{N}\right\rangle}\right) \underline{Z}+\delta_{23} I_{\left\langle\underline{Z},\left.\underline{\sigma}\right|_{N}\right\rangle}\left\langle\underline{Z},\left\langle\underline{Z},\left.\underline{\sigma}\right|_{N}\right\rangle^{-1}\right\rangle \\
& +2\left(\delta_{31}+\delta_{32} I_{\left\langle\underline{Z},\left(\left.\underline{\sigma}\right|_{N}\right)^{2}\right\rangle}\right)\left\langle\underline{Z},\left.\underline{\sigma}\right|_{N}\right\rangle+\delta_{33} I I I_{\left\langle\underline{Z},\left(\left.\underline{\sigma}\right|_{N}\right)^{2}\right\rangle}\left\langle\left.\underline{\sigma}\right|_{N},\left\langle\underline{Z},\left\langle\underline{Z},\left.\underline{\sigma}\right|_{N}\right\rangle^{-1}\right\rangle\right\rangle,
\end{aligned}
$$

with

$$
\delta_{\alpha 1}=\frac{\partial \varphi\left(\mathcal{S}_{G}\right)}{\partial \underline{I}_{\underline{A}_{\alpha}}}, \quad \delta_{\alpha 2}=\frac{\partial \varphi\left(\mathcal{S}_{G}\right)}{\partial I_{\underline{A}_{\alpha}}}, \quad \delta_{\alpha 3}=\frac{\partial \varphi\left(\mathcal{S}_{G}\right)}{\partial I_{\underline{A}_{\alpha}}}, \quad(\alpha=1,2,3),
$$

and

$$
\underline{A}_{1}=\left.\underline{\sigma}\right|_{N}, \quad \underline{A}_{2}=\left\langle\underline{Z},\left.\underline{\sigma}\right|_{N}\right\rangle, \quad \underline{A}_{3}=\left\langle\underline{Z},\left(\left.\underline{\sigma}\right|_{N}\right)^{2}\right\rangle .
$$

The condition of thermodynamic equilibrium for $\underline{d}$ (see (6.5) 1 ) leads to

$$
\left.\delta_{11}\right|_{E}=0,\left.\quad \delta_{12}\right|_{E}=0,\left.\quad \delta_{21}\right|_{E}=0, \quad \text { and }\left.\quad \delta_{22}\right|_{E}=0 .
$$

According to (3.3), the quantity $\frac{1}{2}\left\langle\underline{Z},\left.\underline{\sigma}\right|_{N}\right\rangle$ appearing in (7.16) is defined as the effective dissipative stress and labeled as

$$
\left.\underline{\tilde{\sigma}}\right|_{N}=\frac{1}{2}\left\langle\underline{Z},\left.\underline{\sigma}\right|_{N}\right\rangle \text {. }
$$

Since $\left.\underline{\sigma}\right|_{N}$ or $\left.\underline{\tilde{\sigma}}\right|_{N}$ can be zero, $\underline{d}$, as given in (7.16), is not in general invertible. To guarantee invertibility we must necessarily have [22]

$$
\delta_{13}=0, \quad \delta_{23}=0, \quad \delta_{33}=0,
$$


and so, (7.16) simplifies to

$$
\begin{aligned}
& \underline{d}=\left(\delta_{11}+\delta_{12} I_{\left.\underline{\sigma}\right|_{N}}\right) \underline{I}-\left.\delta_{12} \underline{\sigma}\right|_{N} \\
& +2\left(\delta_{21}+\delta_{22} I_{\left\langle\underline{Z},\left.\underline{\sigma}\right|_{N}\right\rangle}\right) \underline{Z}+\left.4\left(\delta_{31}+\delta_{32} I_{\left\langle\underline{Z},\left(\left.\underline{\sigma}\right|_{N}\right)^{2}\right\rangle}\right) \underline{\tilde{\sigma}}\right|_{N} .
\end{aligned}
$$

The usual form of the dissipative stress of damaged ice consists of the first and the last terms on the right-hand side. $\delta_{11}+\delta_{12} I_{\left.\underline{\sigma}\right|_{N}}$ is then the volumetric (bulk) fluidity (chosen such that $\left.\rho \leq \hat{\rho}\right)$ and $4\left(\delta_{31}+\right.$ $\left.\delta_{32} I_{\left\langle Z,\left(\left.\sigma\right|_{N}\right)^{2}\right\rangle}\right)$ is the shear fluidity. Both depend on stress, damage and temperature.

We still wish to further simplify. So we will suppose that the dissipative flow of damaged ice is independent of the pressure. Such an assumption is tantamount to ignoring the bulk fluidity (Stokes assumption). ${ }^{10}$ The constitutive variables are in this case

$$
\mathcal{S}_{G}^{D}=\left(\hat{\rho}, \underline{\Sigma}, \theta, \underline{Z}, \vec{g},\left.\underline{\sigma}\right|_{N} ^{D}\right) .
$$

Note that we have above assumed that $\underline{d}$ does not depend on $\underline{\Sigma}$ and $\vec{g}$. Considering the property

$$
\underline{d}=\frac{\partial \varphi\left(\mathcal{S}_{G}^{D}\right)}{\left.\partial \underline{\sigma}\right|_{N}}=\left(\frac{\partial \varphi\left(\mathcal{S}_{G}^{D}\right)}{\left.\partial \underline{\sigma}\right|_{N} ^{D}}\right)^{D}
$$

relation (7.22) simplifies to

$$
\underline{d}=-\left.\delta_{12}^{D} \underline{\sigma}\right|_{N} ^{D}+2\left(\delta_{21}^{D}+\delta_{22}^{D} I_{\left\langle\underline{Z},\left.\underline{\sigma}\right|_{N} ^{D}\right\rangle}\right) \underline{Z}^{D}+\left.4\left(\delta_{31}^{D}+\delta_{32}^{D} I_{\left\langle\underline{Z},\left(\left.\underline{\sigma}\right|_{N} ^{D}\right)^{2}\right\rangle}\right) \underline{\tilde{\sigma}}\right|_{N} ^{D},
$$

with

$$
\delta_{12}^{D}=\frac{\partial \varphi\left(\mathcal{S}_{G}^{D}\right)}{\partial \boldsymbol{I}_{\left.\underline{\underline{\sigma}}\right|_{N} ^{D}}}, \quad \delta_{21}^{D}=\frac{\partial \varphi\left(\mathcal{S}_{G}^{D}\right)}{\partial I_{\left\langle\underline{Z},\left.\underline{\sigma}\right|_{N} ^{D}\right\rangle}}, \quad \delta_{22}^{D}=\frac{\partial \varphi\left(\mathcal{S}_{G}^{D}\right)}{\partial I_{\left\langle\underline{Z},\left.\underline{\sigma}\right|_{N} ^{D}\right\rangle}}, \quad \delta_{31}^{D}=\frac{\partial \varphi\left(\mathcal{S}_{G}^{D}\right)}{\partial I_{\left\langle\underline{Z},\left(\left.\underline{\sigma}\right|_{N} ^{D}\right)^{2}\right\rangle}}, \delta_{32}^{D}=\frac{\partial \varphi\left(\mathcal{S}_{G}^{D}\right)}{\partial I_{\left\langle\underline{Z},\left(\left.\underline{\sigma}\right|_{N} ^{D}\right)^{2}\right\rangle}},
$$

and

$$
\left.\underline{\tilde{\sigma}}\right|_{N} ^{D}=\frac{1}{2}\left\langle\underline{Z},\left.\underline{\sigma}\right|_{N} ^{D}\right\rangle^{D}
$$

The thermodynamic condition $(6.5)_{1}$ is satisfied if $\left.\delta_{21}^{D}\right|_{E}=0$ and $\left.\delta_{22}^{D}\right|_{E}=0$. By assuming that the first two terms on the right-hand side of (7.25) are negligible, (7.25) becomes

$$
\left.\underline{d}=\eta_{\mathrm{visc}}^{-1} \underline{\sigma}, \underline{Z}, \theta\right)\left.\underline{\tilde{\sigma}}\right|_{N} ^{D},
$$

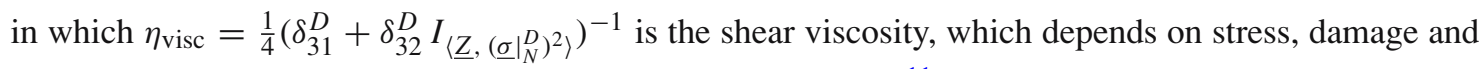
temperature. For damaged ice, Pralong and Funk [14] proposed $^{11}$

$$
\eta_{\mathrm{visc}}(\underline{\sigma}, \underline{Z}, \theta)=A(\theta)^{-1}\left(-I_{\left.\underline{\tilde{\sigma}}\right|_{N} ^{D}}+\kappa_{\sigma}\right)^{\frac{1-n}{2}},
$$

where $A(\theta)>0$ is the fluidity parameter for ice, $n>1$ is the power law exponent and $\kappa_{\sigma}>0$ is a parameter guaranteeing a finite viscosity law [1]. Note the particular form of the effective dissipative stress (7.27), which is a function of the deviatoric part of $\left.\underline{\sigma}\right|_{N}$. Equations (7.28) and (7.29) reduce to the classical Glen-type flow law for $\underline{Z}=\underline{I}$.

The stress $\underline{\sigma}$ appearing in the balance laws (3.4) is determined by the relation $\underline{\sigma}=\left.\underline{\sigma}\right|_{E}+\left.\underline{\sigma}\right|_{N}$. The non-equilibrium stress $\left.\underline{\sigma}\right|_{N}$ is given by (7.28) (or by a more general relation $\underline{d}=\underline{d}\left(\left.\underline{\sigma}\right|_{N}, \ldots\right.$ ) derived in this section) and the equilibrium stress $\left.\underline{\sigma}\right|_{E}$ by (6.10), where $\underline{f}$ is still to be found.

10 Here, this assumption does not mean that the damaged ice is intrinsically density preserving, but that the density variation (the influence of the pressure, respectively) is neglected in the flow law of damaged ice. This is analogous to a Stokes-Kirchhoff gaz.

11 The negative sign in the bracket accounts for the definition of the second invariant given by $(4.20)_{2}$, which is the negative of the second invariant commonly used by glaciologists. 


\subsection{Dynamic function of damage}

Finally we write a constitutive relation for $f\left(\mathcal{S}_{\mathrm{G}}\right)$. Let us first analyze the entropy inequality. Suppose that $\underline{d}$ does not depend on $\vec{g}$. Using (6.2), (6.3) $)_{3},(6.5)_{2},(7.10),(7.29)$, and since $\underline{f}$ is quadratic in $\vec{g}$, one can show that the term

$$
-\rho Y_{i j}\left(f_{i j}-\left.\left.\frac{\partial f_{i j}}{\partial\left(\left.\sigma\right|_{N}\right)_{k l}}\right|_{E} \frac{\partial\left(\left.\sigma\right|_{N}\right)_{k l}}{\partial d_{p q}}\right|_{E} d_{p q}\right),
$$

appearing in inequality (6.11), takes its minimum, namely 0 , at thermodynamic equilibrium, if $\left.\left(\partial f /\left.\partial \underline{\sigma}\right|_{N}\right)\right|_{E}=\underline{0}$ and $f$ is concave with respect to $\left.\underline{\sigma}\right|_{N}$ and $\vec{g}$. The condition of concavity is sufficient but not necessary ((6.7) is always satisfied, not only at equilibrium).

By considering (7.1), inequality (6.11) becomes

$$
\left(\left.\sigma\right|_{N}\right)_{i j} d_{i j}+\frac{\kappa_{i j}}{\theta} g_{i} g_{j}-\rho Y_{i j}\left(f_{i j}-\left.\left.\frac{\partial f_{i j}}{\partial\left(\left.\sigma\right|_{N}\right)_{k l}}\right|_{E} \frac{\partial\left(\left.\sigma\right|_{N}\right)_{k l}}{\partial d_{p q}}\right|_{E} d_{p q}\right) \geq 0 .
$$

With (7.1), (7.28), and (7.29), the first two terms are non-negative and $\left.\underline{d}\right|_{E}=\underline{0}$. The thermodynamic restrictions $\pi \geq 0$ and $\left.\pi\right|_{E}=0$ are therefore always satisfied if $(6.5)_{2}$ is respected, $\left.\left(\partial \underline{f} /\left.\partial \underline{\sigma}\right|_{N}\right)\right|_{E}=\underline{0}$ and $f$ is concave in $\left.\underline{\sigma}\right|_{N}$ and $\vec{g}$.

Now, let us postulate an explicit constitutive relation for the dynamic function of damage as follows

$$
\underline{f}=\varphi_{22} \underline{Z}^{2}+\tilde{\varphi}_{223} \underline{Z}\left\langle\underline{Z},\left.\underline{\sigma}\right|_{N}\right\rangle \underline{Z},
$$

where $\varphi_{22}$ and $\tilde{\varphi}_{223}$ are scalar parameters. We introduce the variable change

$$
\underline{Z}:=(\underline{I}-\underline{D})^{-1}
$$

where $\underline{D}$ is the classical damage tensor. Applying this variable change to (3.12), the objective derivative of $\underline{D}$ reads

$$
\underline{\nabla}=\underline{Z}^{-1} \underline{\nabla}_{\underline{Z}}^{\underline{Z}} \underline{Z}^{-1}+\underline{I} \beta \operatorname{div} \vec{v}-2 \beta \underline{Z}^{-1} \operatorname{div} \vec{v} .
$$

This equation proves the convenience implied by choosing $\beta=0$ as stated earlier. By considering the balance of damage for $\underline{D}$, i.e., $\underline{\nabla}-\underline{f}^{\mathrm{d}}=\underline{0}$, where $\underline{f}^{\mathrm{d}}=\underline{Z}^{-1} \underline{f} \underline{Z}^{-1}$, and setting $\beta=0$, (7.32), with the aid of (7.20), becomes

$$
\underline{f}^{\mathrm{d}}=\varphi_{22} \underline{I}+\left.2 \tilde{\varphi}_{223} \underline{\tilde{\sigma}}\right|_{N} .
$$

Using the spectral decomposition of the symmetric tensor $\left.\underline{\tilde{\sigma}}\right|_{N}$, one obtains

$$
\underline{f}^{\mathrm{d}}=\varphi_{22} \underline{I}+2 \tilde{\varphi}_{223} \sum_{i=1}^{3}\left(\left.\underline{\tilde{\sigma}}\right|_{N}\right)_{i} \vec{\nu}^{(i)} \otimes \vec{v}^{(i)},
$$

with $\vec{v}^{(i)}$ the normalized eigenvectors associated with the eigenvalues $\left(\left.\underline{\tilde{\sigma}}\right|_{N}\right)_{i}$. Considering only the influence of the maximum eigenvalue $\left(\left.\underline{\tilde{\sigma}}\right|_{N}\right)_{1}$ and choosing $\varphi_{22}=f^{1}(1-\gamma)$ and $2 \tilde{\varphi}_{223}\left(\left.\underline{\tilde{\sigma}}\right|_{N}\right)_{1}=f^{1} \gamma$, (7.36) reduces to

$$
\underline{f}^{\mathrm{d}}=f^{1} \underline{R}^{\mathrm{d}},
$$

where $f^{1}$ is a scalar function, $\gamma$ is the anisotropy parameter and

$$
\underline{R}^{\mathrm{d}}=\left[(1-\gamma) \underline{I}+\gamma \vec{v}^{(1)} \otimes \vec{v}^{(1)}\right] .
$$

$f^{1}$ must satisfy condition (5.6), i.e.,

$$
\left.f^{1}\right|_{E}=0,
$$

since $\varphi_{22}$ does so. Equations (7.37) and (7.38) constitute the orthotropic creep damage evolution law by Murakami and Ohno [23]. 

by

The usual form of the function $f^{1}$, when it is applied to describe cracking in creep material, is given

$$
f^{1}=B_{f}\langle\langle\chi\rangle\rangle^{r_{f}} \underline{I}^{k_{f}},
$$

with $B_{f}, r_{f}$ and $k_{f}$ evolution parameters, $\chi$ an equivalent stress (stress measure) and

$$
\left\langle\langle\chi\rangle= \begin{cases}\chi, & \text { if } \quad \chi \geq 0 \\ 0, & \text { if } \quad \chi<0\end{cases}\right.
$$

The equivalent stress is given for example by Hayhurst's criterion [24]

$$
\chi\left(\left.\underline{\tilde{\sigma}}\right|_{N}\right)=\alpha_{\mathrm{H}}\left(\left.\underline{\tilde{\sigma}}\right|_{N}\right)_{1}+\beta_{\mathrm{H}} \sqrt{-\left.3 I I_{\tilde{\tilde{\sigma}}}\right|_{N} ^{D}}+\left(1-\alpha_{\mathrm{H}}-\beta_{\mathrm{H}}\right) I_{\left.\tilde{\tilde{\sigma}}\right|_{N}}
$$

where $\left.\underline{\tilde{\sigma}}\right|_{N}$ is the effective dissipative stress as defined in (7.20). It combines linearly the effective maximum principal stress $\left(\left.\underline{\tilde{\sigma}}\right|_{N}\right)_{1}$, the first invariant $I_{\left.\underline{\tilde{\sigma}}\right|_{N}}$ and the second invariant $I_{\left.\underline{\tilde{\sigma}}\right|_{N} ^{D}}$ of the deviatoric part of the dissipative stress. The parameters $\alpha_{\mathrm{H}}$ and $\beta_{\mathrm{H}}$ characterize the material behavior. Pralong and Funk [14], on the basis of experiments conducted by Gold [25, 26], Duval [27] and Mahrenholtz and $\mathrm{Wu}$ [6], proposed $\alpha_{\mathrm{H}}=0.21$ and $\beta_{\mathrm{H}}=0.63$ for ice close to the melting point and subjected to low deformation rates.

The function $f^{1}$ satisfies condition (7.39). The concavity for $f^{1}$ is given by $r_{f} \geq 1$ and the condition $\left.\left(\partial \underline{f} /\left.\partial \underline{\sigma}\right|_{N}\right)\right|_{E}=\underline{0}$ by $r_{f}>1$. A stress threshold $\sigma_{\text {th }}$ accounting for damage nucleation can be introducing as follows [12]

$$
f^{1}=B_{f}\left\langle\left\langle\chi-\sigma_{\mathrm{th}}\right\rangle\right\rangle^{r_{f}} I_{\underline{Z}^{k}}{ }^{k_{f}} .
$$

$\sigma_{\text {th }}$ was quantified for ice subjected to low deformation rates by Pralong and Funk [14]. This formulation considers the influence of the crack activation energy by simply shifting the function $f^{1}$ to the right. The function (7.43) satisfies also condition (7.39). It presents formal advantages for $\sigma_{\text {th }}>0$. The concavity condition is, as emphasized before, a sufficient condition, for the entropy inequality to be satisfied. If $\underline{f}$ is concave in $\vec{g}$ (for example in the dependence of $B_{f}$ on $\vec{g}$ ), the term (7.30) assumes its minimum, namely zero, at thermodynamic equilibrium for every value of $r_{f}$; that is, the thermodynamic restrictions $(\pi \geq 0$ and $\left.\left.\pi\right|_{E}=0\right)$ are always satisfied.

\subsection{Healing}

The concavity in $\vec{g}$ is a strong restriction for $f$. It forces the term (7.30) to never be negative. Let us now assume that $f$ is not necessary if concave in $\overrightarrow{\vec{g}}$ and rewrite inequality (7.31) as

$$
\left(\left.\sigma\right|_{N}\right)_{i j} d_{i j}+\frac{\kappa_{i j}}{\theta} g_{i} g_{j}-\rho Y_{i j} f_{i j}+\left.\left.\rho Y_{i j} \frac{\partial f_{i j}}{\partial\left(\left.\sigma\right|_{N}\right)_{k l}}\right|_{E} \frac{\partial\left(\left.\sigma\right|_{N}\right)_{k l}}{\partial d_{p q}}\right|_{E} d_{p q} \geq 0 .
$$

The first and the second terms on the left-hand side are related to the energy production supplied by viscous dissipation and heat conduction. They have been shown to be non-negative for the parameterization given by (7.29) and (7.1). The last two terms can be associated with a dissipative crack power. Suppose that $\underline{f}$ grows with $\underline{\sigma}$ near thermodynamic equilibrium, i.e.,

$$
\left.\frac{\partial \underline{f}}{\left.\partial \underline{\sigma}\right|_{N}}\right|_{E} \text { is non-negative definite. }
$$

This is likely satisfied because of the positive feedback observed between damage and stress. By considering (7.28), $\left.\partial \underline{\sigma}\right|_{N} / \partial \underline{d}$ is positive definite. Regarding (7.10), the fourth term in (7.44) is then positive when $\underline{d}$ is negative definite. Therefore, $f$ may be negative definite without violating inequality (7.44). A negative term corresponds to crack healing and a positive term to crack growth. Crack healing is observed in glaciers. It leads to the closure and the localization of crevasses [10]. 
Healing could be considered by subtracting a healing rate function $\underline{f}_{\text {heal }}$ from the dynamic function of damage, in a way similar to Krausz and Krausz [28],

$$
\underline{f}=\underline{f}_{\text {crack }}-\underline{f}_{\text {heal }},
$$

where $\underline{f}_{\text {crack }}=\underline{Z} \underline{f}_{\text {crack }}^{d} \underline{Z}$ is a cracking rate function. $\underline{f}_{\text {crack }}^{d}$ can be approximated, for example, by (7.37). $f_{\text {heal }}$ is, however, not known for ice. In an attempt to model healing, we propose

$$
f_{\text {heal }_{i j}}=C_{f}\left[\left(\left.\sigma\right|_{N}\right)_{k l} d_{k l}+\frac{\kappa_{k l}}{\theta} g_{k} g_{l}\right]\left(\delta_{i j}-Z_{i j}^{-1}\right)^{s_{f}} \text {, }
$$

where $C_{f} \geq 0$ and $s_{f}>0$ are the parameters of the healing function. This function depends on the viscous dissipation and on the heat conduction which provide the energy of healing. The dependence on $\underline{Z}$ leads to $\underline{f} \underline{\text { heal }}_{\text {e }}=\underline{0}$ for $\underline{Z}=\underline{I}$ (no damage) and prevents damage to decrease for virgin ice.

Let us now assume the parametrization (7.43) for $\underline{f}_{\text {crack }}$ and suppose that $\underline{f}_{\text {crack }}$ is concave in $\vec{g} .{ }^{12}$ Let us, moreover, assume that $\underline{d}$ does not depend on $\vec{g}$, that $n \geq 3$ and that $\sigma_{\text {th }}>0$. With the help of (7.10) and (7.29) and since $f$ is quadratic in $\vec{g}, \underline{\kappa}$ is positive definite, $\underline{\kappa}$ does not depend on $\left.\underline{\sigma}\right|_{N}$ and $\vec{g}$, and (6.5) 1 is fulfilled, the residual entropy production achieves its minimum, namely 0 , at equilibrium if

$$
1+C_{f} \rho Y_{i j}\left(\delta_{i j}-Z_{i j}^{-1}\right)^{S_{f}} \geq 0
$$

The thermodynamic restrictions $\pi \geq 0$ and $\left.\pi\right|_{E}=0$ are in this case always satisfied.

\section{Numerical example}

In order to present the effect of damage induced anisotropy on the flow of ice, we apply the above damage model to the case of a plane gravity driven flow down an inclined plane surface.

\subsection{Assumptions}

For this computation, the following assumptions are made:

1. Constant temperature (isothermal condition) $(\partial \theta=0)$

2. The elastic stresses are small as compared to the viscous stresses $(\underline{\Sigma} \rightarrow \underline{0}$; see (7.6) and (7.8))

3. Acceleration terms are neglected in the momentum balance $(\rho \dot{\vec{v}} \rightarrow 0)$

4. Plane strain flow $\left(\partial / \partial y=0\right.$ and $v_{y}=0$; flow in the $x-z$ plane $)$

5. Parallel sided slab subject to zero longitudinal changes $(\partial / \partial x=0)$ and steady state geometry

6. Density variations are neglected for the ice flow $(\partial \rho=0)$

7. No healing $\left(C_{f}=0\right)$

To model glaciers or ice caps in two dimensions, the first four assumptions are usually made in glaciology. The parallel sided slab hypothesis is assumed for simplicity. Since a parallel sided slab deforms in shearing, the cracks are assumed not to open. The density variations of the damaged ice are therefore inactive and corresponding terms vanish. Finally, healing is not considered since proposal (7.47) has not been validated.

12 This condition might be too restrictive (see Sect. 7.4) 


\subsection{Model}

Hypothesis 1 implies that the energy balance need not be considered. Hypothesis 3 reduces the momentum balance to $\operatorname{div} \underline{\sigma}+\rho \vec{b}=\overrightarrow{0}$. From assumption 2 and (7.8), it follows that $\underline{Y}=\underline{0}$ and therefore with (6.10) that $\underline{\sigma}=\left.\underline{\sigma}\right|_{N}$. With hypothesis 6 , the mass balance reduces to $\operatorname{div} \vec{v}=\overrightarrow{\overrightarrow{0}}$ and relation (7.28) can be used to model the dissipative strain rate. Using identity $\underline{\sigma}=\left.\underline{\sigma}\right|_{N}$ and (7.27), relation (7.28) becomes

$$
\left(\underline{\underline{Z}} \underline{\sigma}^{D}\right)^{D}=\eta_{\text {visc }}(\underline{\sigma}, \underline{Z}, \theta) \underline{d},
$$

with $\underline{\underline{Z}}$ described by (3.2) and $\eta_{\text {visc }}$ given in (7.29). $\underline{\underline{Z}}$ is here a function of $\underline{Z}$. This dependence is explicitly proposed in [13]. By considering the relation $\underline{\sigma}^{D}=\underline{\sigma}+p \underline{I}$ with $p$ the pressure, (8.1) becomes

$$
\underline{\sigma}=-p_{1} \underline{I}-p_{2} \underline{\underline{Z}}^{-1} \underline{I}+\eta_{\mathrm{visc}}(\underline{\sigma}, \underline{Z}, \theta) \underline{\underline{Z}}^{-1} \underline{d},
$$

where $p_{1}$ and $p_{2}$ are two distinct pressures resulting from the two deviatoric indices of (7.27). They reduce to $p=p_{1}+p_{2} / Z$ for isotropic damage only. To bypass this problem, one could express the dissipative deformation by using an alternative dissipation potential

$$
\left.\underline{\sigma}\right|_{N}=\frac{\partial \tilde{\varphi}\left(\mathcal{S}^{\mathrm{z}}\right)}{\partial \underline{d}}
$$

with $\mathcal{S}^{\mathrm{Z}}=\left(\hat{\rho}, \underline{B}, \theta, \underline{Z}^{-1}, \vec{g}, \underline{d}\right) .{ }^{13}$ Assuming that $\tilde{\varphi}$ is a function of $\theta, \underline{Z}^{-1}$ and $\underline{d}$ only, omitting explicit terms in $\underline{Z}^{-2}, \underline{d}^{2}$ and of higher order, guaranteeing invertibility of $\left.\underline{\sigma}\right|_{N}$ and assuming that damaged ice is density preserving, we found

$$
\left.\underline{\sigma}\right|_{N} ^{D}=-\tilde{\delta}_{12}^{D} \underline{d}+2\left(\tilde{\delta}_{21}^{D}+\tilde{\delta}_{22}^{D} I_{\left\langle\underline{Z}^{-1}, \underline{d}\right\rangle}\right) \underline{Z}^{-D}+4\left(\tilde{\delta}_{31}^{D}+\tilde{\delta}_{32}^{D} I_{\left\langle\underline{Z}^{-1}, \underline{d}^{2}\right\rangle}\right) \underline{\tilde{d}}^{D},
$$

with

$$
\tilde{\delta}_{12}^{D}=\frac{\partial \tilde{\varphi}\left(\mathcal{S}^{\mathrm{z}}\right)}{\partial \boldsymbol{I I}_{\underline{d}}}, \quad \tilde{\delta}_{21}^{D}=\frac{\partial \tilde{\varphi}\left(\mathcal{S}^{\mathrm{z}}\right)}{\partial I_{\left\langle\underline{Z}^{-1}, \underline{d}\right\rangle}}, \quad \tilde{\delta}_{22}^{D}=\frac{\partial \tilde{\varphi}\left(\mathcal{S}^{\mathrm{z}}\right)}{\partial I_{\left\langle\underline{Z}^{-1}, \underline{d}\right\rangle}}, \quad \tilde{\delta}_{31}^{D}=\frac{\partial \tilde{\varphi}\left(\mathcal{S}^{\mathrm{z}}\right)}{\partial I_{\left\langle\underline{Z}^{-1}, \underline{d}^{2}\right\rangle}}, \quad \tilde{\delta}_{32}^{D}=\frac{\partial \tilde{\varphi}\left(\mathcal{S}^{\mathrm{z}}\right)}{\partial I_{\left\langle\underline{Z}^{-1}, \underline{d}^{2}\right\rangle}},
$$

and

$$
\underline{\tilde{d}}^{D}=\frac{1}{2}\left\langle\underline{Z}^{-1}, \underline{d}\right\rangle^{D} .
$$

By neglecting the first two terms on the right-hand side of (8.4), using the identity $\underline{\sigma}=\left.\underline{\sigma}\right|_{N}$ and considering the relation $\underline{\sigma}^{D}=\underline{\sigma}+p \underline{I}$, the dissipative stress becomes

$$
\underline{\sigma}=-p \underline{I}+\eta_{\mathrm{visc}}(\underline{d}, \underline{Z}, \theta) \underline{\tilde{d}}^{D},
$$

where $\eta_{\mathrm{visc}}(\underline{d}, \underline{Z}, \theta)=4\left(\tilde{\delta}_{31}^{D}+\tilde{\delta}_{32}^{D} I_{\left\langle\underline{Z}^{-1}, \underline{d}^{2}\right\rangle}\right)$ can be expressed as

$$
\eta_{\mathrm{visc}}(\underline{d}, \theta)=A(\theta)^{-\frac{1}{n}}\left(-I_{\underline{d}}+\kappa_{d}\right)^{\frac{1-n}{2 n}}
$$

with the finite viscosity parameter $\kappa_{d}>0$. For isotropic damage and if $\kappa_{\sigma}, \kappa_{d} \rightarrow 0,(8.2)$ and (8.7) can directly be transformed to one another.

Hypothesis 4 leads to $d_{x y}=d_{y z}=0$. With (8.2) or (8.7) and the proposed dynamic function of damage (relations (7.37) and (7.38)), it can be shown that $\sigma_{x y}=\sigma_{y z}=0$ and $Z_{x y}=Z_{y z}=0$. The stress and the damage tensors both can therefore be expressed by a plane representation as the strain rate. We define a Cartesian frame, in which the $x$ - and $z$-axes are parallel and normal to the slope of the slab. $z=0$

13 The dependence on $\underline{Z}^{-1}$, instead of $\underline{Z}$, is universally valid, since $\underline{Z}^{-1}$ can be regarded as a function of $\underline{Z}$. 


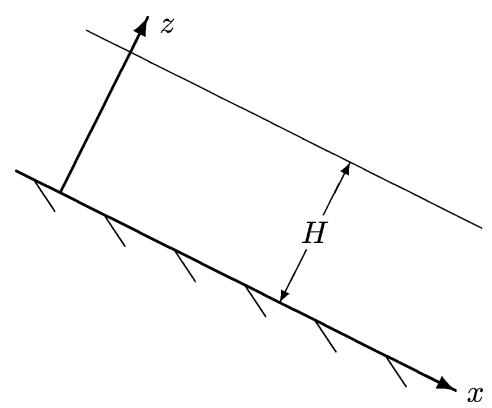

Fig. 1 Geometry of the glacier, as used in the numerical example

defines the bed and $z=H$ the free surface of the glacier slab, where $H$ is the ice thickness (Fig. 1). The boundary conditions then are

$$
\begin{aligned}
& v_{x}(z=0)=v_{x 0}, v_{z}(z=0)=0, \\
& p(z=H)=0, \quad \sigma_{x z}(z=H)=0 .
\end{aligned}
$$

With assumptions 4 and 5, (8.7) and (8.9), the balance laws, integrated over $z$, reduce to

$$
\begin{aligned}
& v_{z}=0, \\
& \frac{1}{2} \eta_{\mathrm{visc}}(\underline{d}, \theta)\left[1-\frac{1}{2}\left(D_{x x}+D_{z z}\right)\right] \frac{\partial v_{x}}{\partial z}=\rho b_{x}(H-z), \\
& p=-\rho b_{z}(H-z)-\frac{1}{3} \rho b_{x}(H-z) D_{x z}\left[1-\frac{1}{2}\left(D_{x x}+D_{z z}\right)\right]^{-1} .
\end{aligned}
$$

The first equation results from the balance of mass and the next two from the balance of linear momentum in the $x$ and $z$ directions, respectively. Relations (8.8) and (8.10) 2 furnish the differential equation for $v_{x}$, for which the boundary condition is $v_{x}(z=0)=v_{x 0}$. The pressure is given by the analytic solution $(8.10)_{3}$. The differential equations for damage read

$$
\begin{aligned}
& \frac{\partial D_{x x}}{\partial t}-\frac{\partial v_{x}}{\partial z} D_{x z}=f_{x x}^{d}, \\
& \frac{\partial D_{y y}}{\partial t}=f_{y y}^{d}, \\
& \frac{\partial D_{z z}}{\partial t}+\frac{\partial v_{x}}{\partial z} D_{x z}=f_{z z}^{d}, \\
& \frac{\partial D_{x z}}{\partial t}+\frac{1}{2} \frac{\partial v_{x}}{\partial z} D_{x x}-\frac{1}{2} \frac{\partial v_{x}}{\partial z} D_{z z}=f_{x z}^{d},
\end{aligned}
$$

with $\underline{f}^{d}$ given by (7.37), (7.38), (7.41), (7.42) and (7.43). The evolution of $D_{y y}$ must be calculated, since $D_{y y}$ appears in $f^{d}$. The initial conditions are given by $\underline{D}=\underline{0}$. Physically, the eigenvalues of $\underline{D}$ must be bounded between 0 (no damage) and 1 (full damage); for $\underline{Z}$, between 1 and $\infty$, respectively. The equations are integrated by using the finite elements method (Femlab code). The implementation is performed in one dimension. A decoupling algorithm is used (e.g., [29]); that is, the damage equations and the flow equations are solved separately. At each time step, the damage problem is solved first (in space with Lagrange elements of order two and in time with a standard Runge-Kutta scheme). The velocity and pressure are approximated with the results from the previous time step. The non-linear equation of the flow model is then solved by iteration on the viscosity. The value of the parameters used in the model are listed in Table 1. 
Table 1 Values of the parameters used in the model. The damage and flow parameters are given by [14]. The thickness and the slope of the slab reproduce approximatively the shape of a hanging glacier

\begin{tabular}{ll}
\hline$A=6.8 \times 10^{-24}\left(\mathrm{~Pa}^{-n} \mathrm{~s}^{-1}\right)$ & $v_{x 0}=0$ \\
$b_{x}=9.81 \sin \left(40^{\circ}\right)\left(\mathrm{m} \mathrm{s}^{-2}\right)$ & $\alpha_{\mathrm{H}}=0.21$ \\
$b_{z}=9.81 \cos \left(40^{\circ}\right)\left(\mathrm{m} \mathrm{s}^{-2}\right)$ & $\beta_{\mathrm{H}}=0.63$ \\
$B_{f}=1.7 \times 10^{-9}\left(\mathrm{~Pa}^{-r} \mathrm{~s}^{-1}\right)$ & $\gamma=$ free \\
$H=30(\mathrm{~m})$ & $\kappa_{\underline{d}}=6 \times 10^{-21}\left(\mathrm{~s}^{-2}\right)$ \\
$k_{f}=k_{1}\left(\left|I_{\left.\underline{\sigma}\right|_{N}}\right|\right)^{0.5}$ & $C_{f}=0$ \\
$k_{1}=3.75 \times 10^{-3}\left(\mathrm{~Pa}^{-\frac{1}{2}}\right)$ & $\rho=910\left(\mathrm{~kg} \mathrm{~m}^{3}\right)$ \\
$n=3$ & $\sigma_{\mathrm{th}}=3.3 \times 10^{5}(\mathrm{~Pa})$ \\
$r_{f}=0.43$ & \\
\hline
\end{tabular}
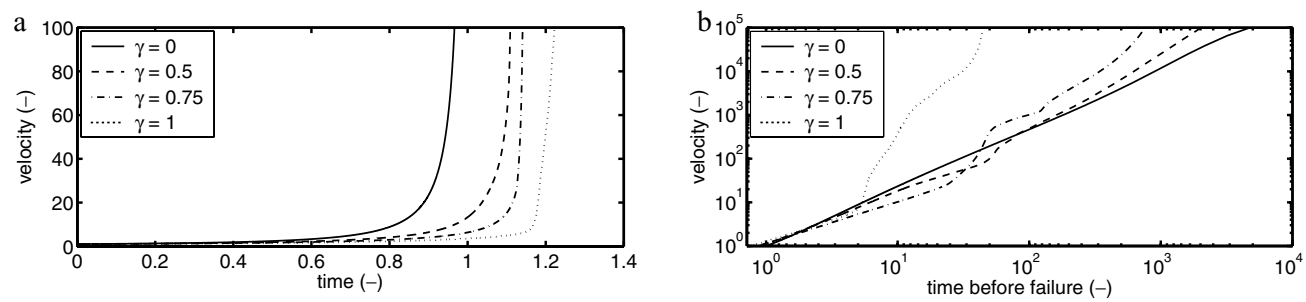

Fig. 2 Relative surface velocity (relative to the initial surface velocity) of the parallel sided slab as a function of the relative time (relative to the time of failure $t_{\mathrm{f}}=46.1$ days of the isotropic case) for different values of the anisotropy parameter $\gamma$. a) linear representation, b) logarithmic representation with the time before failure defined as $t_{\mathrm{f}}-t$

\subsection{Results}

Figure 2a shows the evolution of the surface velocity of the ice slab as a function of time for different values of the anisotropy parameter $\gamma$ (see (7.38)). The velocity reaches a finite time singularity; that is, it increases indefinitely (forming a singularity), while the time $t_{\mathrm{f}}$ corresponding to the infinite velocity (time of failure) is finite. The time of failure of the slab depends on the choice of $\gamma$. Finite-time singularities can be described by Voight's differential equation [30], which models the temporal evolution of a critical quantity (here the displacement of a material point at $z=H$ ). The time integration of Voight's equation leads to [30]

$$
v_{x}(t, z=H)=a_{\Omega}\left(t_{\mathrm{f}}-t\right)^{-m_{\Omega}},
$$

with $a_{\Omega}$ and $m_{\Omega}$ the parameters characterizing the acceleration of the unstable ice chunk. Figure $2 \mathrm{~b}$ shows the logarithm of the velocity as a function of the logarithm of the time $t_{\mathrm{f}}-t$. In this representation, (8.12) is described by a straight line.

Figures 3 presents the evolution of the damage for different anisotropies near the glacier bed (at $z=H / 100$ ). The vorticity of the flow induces a rotation of the damage tensor (the rotation is modeled by the Jaumann derivative). For anisotropic damage, the different components of the damage tensor are thus oscillating around the values of the diagonalized damage tensor. The rotation of the flow increases with the velocity gradient and leads to accelerating oscillations of the damage components.

Figure 4a depicts the distribution of damage along the vertical axis on the verge of failure (at $t=0.98 t_{\mathrm{f}}$ ) for different anisotropies. The kinematics of the damage accumulation is delayed when $z$ increases, due to the decrease of $\chi$ (see (7.43)) with increasing $z$. The damage evolution observed along the $t$-axis in Fig. 3 appears here along the $z$-axis. Above $z \approx 0.4$ no damage develops, because of the stress threshold $\sigma_{\text {th }}$ introduced in the dynamic function of damage (7.43).

The term $\frac{1}{2}\left(D_{x x}+D_{z z}\right)$, appearing in $(8.10)_{2}$ and controlling the surface velocity, does not oscillate, since the oscillating contributions of $D_{x x}$ and $D_{z z}$ vanish (Fig. $4 \mathrm{~b}$ ). The prominent discontinuity observed during the evolution of $\frac{1}{2}\left(D_{x x}+D_{z z}\right)$ for $\gamma=1$ coincides with the period when $D_{y y} \neq 0$ (Fig. $3 \mathrm{~d}$ and f). 

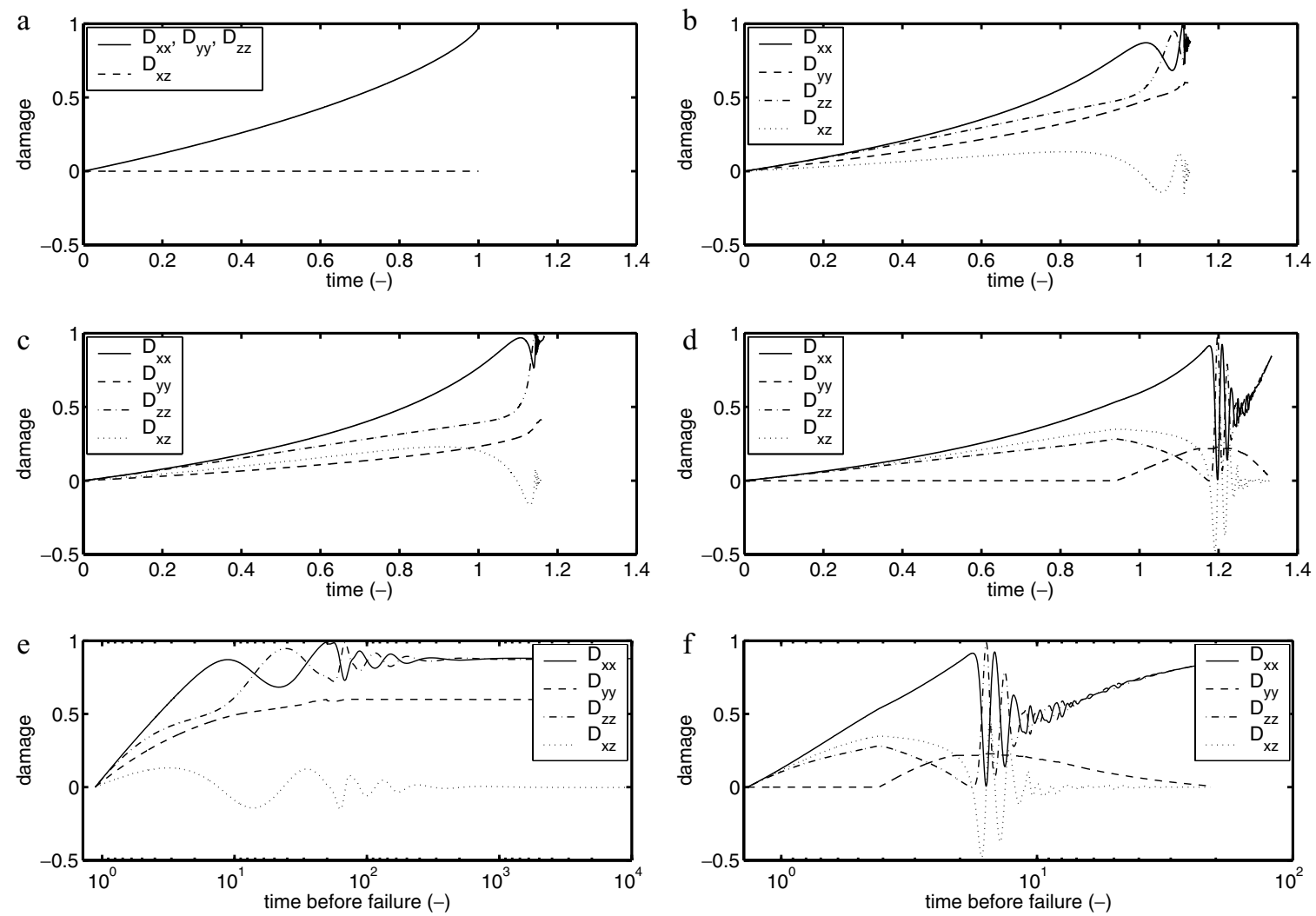

Fig. 3 Evolution of the non-zero components of the damage tensor $D$ as a function of the relative time (relative to the time of failure of the isotropic case) at $z=H / 100$. Damage versus time for different anisotropies: a) $\gamma=0$, b) $\gamma=0.5$, c) $\gamma=0.75$, d) $\gamma=1$ and damage versus logarithmic time before failure (defined as $t_{\mathrm{f}}-t$ ) for e) $\gamma=0.5$ and f) $\gamma=1$
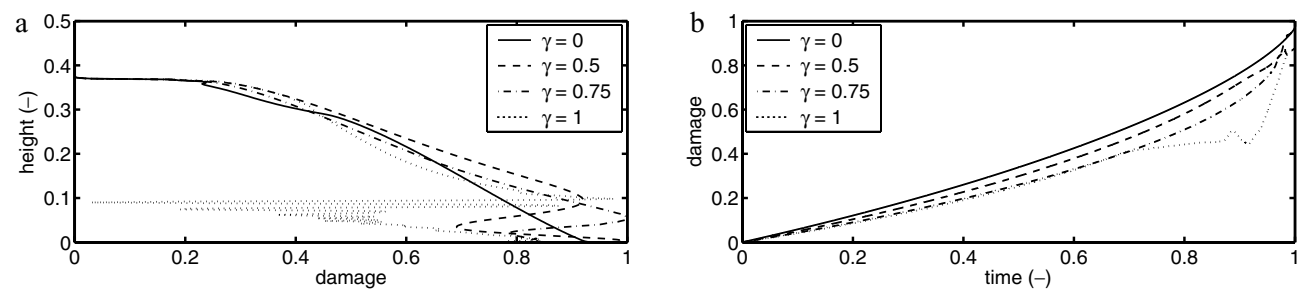

Fig. 4 a) Distribution of $D_{x x}$ as a function of the relative height of the ice slab at $t=0.98 t_{\mathrm{f}}$ for different anisotropies. b) Temporal evolution of $\frac{1}{2}\left(D_{x x}+D_{z z}\right)$ for different anisotropies at $z=H / 100$. For each curve, the time is normed with the failure time of the corresponding anisotropy

During that period, $R_{y y}^{\mathrm{d}}$ (see (7.37)) is first positive and then negative ${ }^{14}$ forcing $D_{y y}$ to increase and decrease, respectively. These variations of $R_{y y}^{\mathrm{d}}$ are due to the rotation of the principal eigenvector of $\left.\underline{\tilde{\sigma}}\right|_{N}$. At the same time, the terms $R_{x x}^{\mathrm{d}}$ and $R_{z z}^{\mathrm{d}}$ are also affected by the rotation of the principal eigenvector of $\left.\underline{\tilde{\sigma}}\right|_{N}$ and induce the discontinuity of $\frac{1}{2}\left(D_{x x}+D_{z z}\right)$. Similar disturbances of $\frac{1}{2}\left(D_{x x}+D_{z z}\right)\left(D_{y y}\right.$, respectively) occur also for $\gamma=0.5$ and $\gamma=0.75$. They are smaller and happen closer to the failure time. No disturbances appear for isotropic ice, since the direction of the principal eigenvector of $\left.\underline{\tilde{\sigma}}\right|_{N}$ is not

14 The negative value of $R_{y y}^{\mathrm{d}}$ (and $f_{y y}^{\mathrm{d}}$, respectively) is due to the change of direction of the damage accumulation. It does not correspond to healing. 
considered in the dynamic function of damage. For anisotropic damage, the disturbances of $\frac{1}{2}\left(D_{x x}+D_{z z}\right)$ influence the surface velocity. The result is that the surface velocity can be described by (8.12) only until the disturbances appear. Field measurements, carried out on unstable ice masses with a geometry similar to that of the model, show that the velocity can adequately be described by $(8.12)$ [31, 32]. However, these measurements do not cover more than the first order of magnitude of the surface velocity increase and therefore cannot validate the results of this numerical example.

\section{Conclusions}

The thermodynamic restrictions to the parameterization of compressible viscoelastic materials with orthotropic damage have been derived. Using these restrictions, a model for ice has been proposed. The ice is considered as a viscoelastic solid where the viscosity and elasticity are influenced by orthotropic damage. The elastic and damage effects have been derived, such that, if they are ignored, the classical Glen-type flow law, which describes the viscous deformations of ice, can be restored. The strain (stress, respectively) equivalence principle, which is commonly used in continuum damage mechanics, emerges here from the assumption that the strain rate (stress, respectively) can be derived from a dissipative potential depending on the damage and the stress (strain, respectively). The objective time derivative of the damage tensor has been determined to be the Jaumann derivative. The damage accumulation law by Murakami appears to be a particular form of the isotropic expansion of our choice of constitutive variables. Its thermodynamic restrictions are presented. Damage healing, which is crucial for the dynamics of crevassed glaciers, is not known for ice. A damage healing function, which satisfies the thermodynamic restrictions, has been proposed. It has, however, not be validated by measurements.

Acknowledgements The authors want to thank two anonymous referees for their comments, which improved the paper and Ondrej Papes for valuable discussions regarding spatially objective time derivatives. This work was partially accomplished within the GLACIORISK project (Survey and Prevention of Extreme Glaciological Hazards in European Mountainous Regions) (Grant No EVG1-2000-00018 and BBW 00.0209-1).

\section{References}

1. Hutter, K.: Theoretical glaciology; material science of ice and the mechanics of glaciers and ice sheets. D. Reidel Publishing Company Tokyo (1983)

2. Sinha, N.-K.: Kinetics of microcracking and dilatation in polycrystalline ice. In: IUTAM/IAHR Symposium on IceStructure Interaction. 69-87 (1991)

3. Xiao, J., Jordaan, I.-J.: Application of damage mechanics to ice failure in compression. Cold Regions Science and Technology. 24(3), 305-322 (1996)

4. Singh, S.-K., Jordaan, I.-J.: Constitutive behaviour of crushed ice. International Journal of Fracture. 97(1-4), 171-187 (1999)

5. Szyszkowski, W., Glockner, P.-G.: On a multiaxial constitutive law for ice. Mechanics of Materials. 5(1), 49-71 (1986)

6. Mahrenholtz, O., Wu, Z.: Determination of creep damage parameters for polycrystalline ice. Advances in Ice Technology. 181-192 (1992)

7. Kachanov, L.-M.: Rupture time under creep conditions (trans. from Russian, 1957). International Journal of Fracture. 97(1-4), xi-xviii (1999)

8. Schapery, R.-A.: Models for the deformation behavior of viscoelastic media with distributed damage and their applicability to ice. In: Sympsium on Ice-Structure Interaction. 191-230 (1991)

9. Melanson, P.-M., Jordaan, I.-J., Meglis, I.-L.: Modelling of damage in ice. In: Proceeding of the 14th International Symposium on Ice. vol. 2, 979-988 (1998)

10. Pralong, A., Funk, M., Lüthi, M.-P.: A description of crevasse formation using continuum damage mechanics. Annals of Glaciology. 37, 77-82 (2003)

11. Weiss, J., Gay, M.: Fracturing of ice under compression creep as revealed by a multifractal analysis. Journal of Geophysical Research. 103(B10), 24005-24016 (1998)

12. Lemaitre, J.: A Course on Damage Mechanics. Springer Berlin (1992)

13. Wu, Z., Mahrenholtz, O.: Creep and creep damage of polycrystalline ice under multi-axial variable loading. In: Proceedings of the 12th International Conference on Offshore Mechanics and Arctic Engineering. vol. 4, 1-10 (1993)

14. Pralong, A., Funk, M.: Dynamic damage model of crevasse opening and application to glacier calving. Journal of Geophysical Research 110, 301-309 (doi: 1029/2004 33003104, 2005).

15. Holzapfel, G.-A.: Nonlinear Solid Mechanics, a Continuum Approach for Engineering. John Wiley \& Sons, LTD, Chichester (2000) 
16. Liu, I.-S.: Method of Lagrange multipliers for exploitation of entropy principle. Archive for Rational Mechanics and Analysis. 46(2), 131-148 (1972)

17. Svendsen, B., Hutter, K., Laloui, L.: Constitutive models for granular materials including quasi-static frictional behaviour: Toward a thermodynamic theory of plasticity. Continuum Mechanics and Thermodynamics. 11(4), 263-275 (1999)

18. Hutter, K., Jöhnk, K.: Continuum Methods of Physical Modeling : Continuum Mechanics, Dimensional Analysis, Turbulence. Springer, Berlin (2004)

19. Liu, I.-S.: On entropy flux heat flux relation in thermodynamics with lagrange multipliers. Continuum Mechanics and Thermodynamics. 8(4), 247-256 (1996)

20. Betten, J.: Tensorrechnung für Ingenieure. B. G. Teubner, Stuttgart (1987)

21. Lemaitre, J., Desmorat, R., Sauzay, M.: Anisotropic damage law of evolution. European Journal of Mechanics ASolids. 19(2), 187-208 (2000)

22. Svendsen, B., Hutter, K.: A continuum approach for modelling induced anisotropy in glaciers and ice sheets. Annals of Glaciology. 23, 262-269 (1996)

23. Murakami, S., Ohno, N.: A continuum theory of creep and creep damage. In: Proc. of the 3rd IUTAM Symposium on Creep in Structures. 422-444 (1980)

24. Hayhurst, D.-R.: Creep-rupture under multi-axial states of stress. Journal of the Mechanics and Physics of Solids. 20(6):381-390 (1972)

25. Gold, L.-W.: Time to formation of first cracks in ice. In: Physics of Snow and Ice, International Conference on low Temperature Science. vol. 1(Part 1), 359-370 (1967)

26. Gold, L.-W.: The failure process in columnar-grained ice. Technical Report 369, Division of Building Research, NRC Ottawa (1972)

27. Duval, P.: Creep and fabrics of polycrystalline ice under shear and compression. Journal of Glaciology. 27(95), 129$140(1981)$

28. Krausz, A.-S., Krausz, K.: Fracture Kinetics of Crack Growth. Kluwer Academic Publishers, Dordrecht (1988)

29. Picasso, M., Rappaz, M.-J., Reist, A., Funk, M., Blatter, H.: Numerical simulation of the motion of a two dimensional glacier. International Journal for Numerical Methods in Engineering. 60(5), 995-1009 (2004)

30. Voight, B.: A method for prediction of volcanic-eruptions. Nature. 332(6160), 125-130 (1988)

31. Röthlisberger, H.: Eislawinen und Ausbrüche von Gletscherseen. In: Gletscher und Klima - glaciers et climat, Jahrbuch der Schweizerischen Naturforschenden Gesellschaft, wissenschaftlicher Teil 1978. 170-212 (1981)

32. Lüthi, M.: Instability in glacial systems. In: Milestones in Physical Glaciology: From the Pioneers to a Modern Science. VAW, ETH Zürich. vol. 180, 63-70 (2003) 